\title{
Partial information or facilitation? Different interpretations of results from speed-accuracy decomposition
}

\author{
RITSKE DE JONG \\ Ohio State University, Columbus, Ohio
}

\begin{abstract}
The speed-accuracy decomposition technique was developed by Meyer, Irwin, Osman, and Kounios (1988) to examine the time course of information processing. The technique allows for the estimation of the accuracy of guesses that are induced by the presentation of a response signal on a proportion of trials. Estimated guessing accuracy has been found to be above chance and to increase as time of guessing increases, suggesting that guesses are based on partial information that has accumulated prior to a response decision (sophisticated guesses). In this paper, a different interpretation of these data is presented. Results suggest that response signals may enhance the speed of regular processes, thereby violating the temporal-independence assumption that underlies the decomposition technique. As shown by Monte Carlo simulations, such facilitating effects of response signals can explain the results from the decomposition technique at least in part and possibly in full, even when guesses are actually at chance accuracy (pure guesses). The pure-guess model was supported by the results from an experiment designed to test between the alternative interpretations. These results point to the need for great caution in the attempt to infer the time course of information processing from guessing accuracies as estimated by the speed-accuracy decomposition technique.
\end{abstract}

A major challenge for students of human information processing is to infer the organization and dynamics of covert psychological processes from the measured effects of experimental manipulation on overt behavior. Available inferential techniques, such as the additive factor method (Sternberg, 1969), have been, and continue to be, highly useful in this respect; but their inferential power is limited, and the validity of the assumptions on which they are based is not generally agreed on (Meyer, Irwin, Osman, \& Kounios, 1988; Miller, 1988). What is needed in order to meet the challenge is the ability to assess the intermediate states of the processing system during the perception and evaluation of input information and the selection, programming, and execution of appropriate courses of action. Meyer et al. (1988) have recently developed a technique that may provide an important tool for the assessment of such intermediate states.

This technique involves two randomly intermixed types of trials. On one type of trial, subjects are presented with a response signal that requires them to respond immediately, even if primary-task processing is still in progress (signal trials); on the other type of trial, no response sig-

This work was supported by Grant 1R01-MH41445 from the National Institute of Mental Health. I would like to thank Mike Coles, Bill Gehring, Art Kramer, Gordon Logan, Jeff Miller, and James Townsend for helpful comments on earlier drafts of this manuscript. Correspondence concerning this article should be addressed to Ritske De Jong, Department of Psychology, Ohio State University, 142 Townshend Hall, 1885 Neil Avenue, Columbus, OH 43210. nal is presented, and subjects respond normally (regular trials). It is assumed that on signal trials regular processes and guessing processes race against each other in such a way that whichever set of processes finishes first will determine the response. Thus, responses on signal trials are assumed to be a mixture of relatively fast regular responses, which are based on complete information, and informed guesses, which are based on whatever partial processing results are available to the subject at the time of guessing. This mixture can then be decomposed, and both the reaction time distribution and the accuracy of the guess responses can be estimated (see Meyer et al., 1988, for details).

This new technique, referred to as the speed-accuracy decomposition technique, has been applied to a number of tasks such as lexical decision (Meyer et al., 1988), sentence verification (Kounios, Osman, \& Meyer, 1987), and study-test recognition (Ratcliff, 1988). A common finding in these studies has been that the estimated accuracy of guesses is well above chance and increases as a function of the interval between the test stimulus and the response signal. The first result suggests that the guesses are based on partial information that accumulates prior to a normal response decision, and the second result suggests that the guessing accuracy function reflects the time course of the accumulation of partial processing products.

Whereas some uncertainty remains as to whether partial information accumulates continuously (Ratcliff, 1988) or in a small number of discrete steps (see, e.g., Meyer et al., 1988), investigators appear to agree on this in- 
terpretation of the results obtained with the speed-accuracy decomposition technique. In this article, I will argue that these results can also be interpreted in a way that does not require the assumption that guesses are based on partial processing output. If this argument is correct, it should suggest that inferences from the accuracy of guesses, as estimated by the decomposition technique, to hypothetical intermediate processing states should be made with great caution and that, under some circumstances, such inferences cannot usefully be made at all.

\section{The Temporal-Independence Assumption}

In the present paper, I will focus on the consequences of possible violations of one of the basic assumptions underlying the speed-accuracy decomposition technique, which Meyer et al. (1988) have referred to as the temporal-independence assumption. This assumption actually involves two separate assumptions. First, regular processes are assumed to proceed in the same way, regardless of whether or not a response signal is presented (signal independence). Second, when a response signal is presented, the durations of the guessing and the regular processes are assumed to be stochastically independent (stochastic independence).

Meyer et al. (1988) discussed two analyses based on the estimated guessing-duration distribution, which they considered to be stringent tests of the temporalindependence assumption. First, they argued that invariance of the estimated guessing-duration distribution with type of test stimulus (stimulus-type invariance) would constitute evidence for the validity of the temporalindependence assumption. However, stimulus-type invariance can only demonstrate that possible effects of violations of temporal independence do not interact with stimulus type-not, in general, that such effects are altogether absent. The fact that stimulus-type invariant guessing-duration distributions have been obtained in all the studies in which the decomposition technique has been used therefore provides suggestive but not conclusive evidence for temporal independence.

The second analysis proposed by Meyer et al. (1988) concerns invariance of the guessing-duration distribution with response signal lag (signal-lag invariance). There is evidence that signal-lag invariance may not always hold. In some of the experiments reported by Meyer et al. (Experiments 1, 3, and 5; see Tables 3, 9, and 13), mean estimated guessing duration tended to decrease as response signal lag increased. Such effects of signal lag on mean guessing duration are also evident in the results reported by Ratcliff (1988). ${ }^{1}$ These findings would seem to suggest that the temporal-independence assumption may not be strictly valid. However, they can also be reasonably interpreted in a way that would leave this assumption intact. In this interpretation, the test stimulus is viewed as functioning, in part, as a warning stimulus for the response signal, with the response signal lag viewed as the analogue of the foreperiod in warned simple reaction time tasks. With variable foreperiods, simple reaction times are typically longer at the shorter foreperiods (for a review, see Niemi \& Naatanen, 1981). This alternative interpretation may serve to save the temporalindependence assumption, but it also limits the usefulness of the signal-lag invariance test of this assumption.

The speed-accuracy decomposition technique thus appears to have passed some tests of its inferential logic reasonably well, but the adequacy of these tests remains uncertain. Because the technique is a potentially important new tool in the study of human information processing, it is important to test its validity more rigorously and to explore possible alternative explanations of the results that it has produced. The present paper presents such a critical examination of the technique.

The remainder of this paper is organized as follows. I will begin by discussing the possibility that some of the processes involved in primary-task performance may be facilitated by the presentation of the response signal, resulting in a violation of signal independence. I will show how such facilitation can account, at least qualitatively, for the increase of estimated guessing accuracy and the decrease of estimated mean guessing duration with signal lag, even if guesses are actually at chance accuracy. Next, I will present the results of an experiment designed for the purpose of investigating whether such facilitation might actually be operative in response-signal paradigms. Results will then be presented from two Monte Carlo simulations in which quantitative predictions of the proposed alternative interpretation of the results from the speed-accuracy decomposition technique will be compared in detail with two representative data sets. Finally, two differential predictions will be derived from the two alternative interpretations, and results will be presented from an experiment in which these interpretations were tested.

\section{The Pure-Guess Model}

It has long been known that simple reactions to a combined visual-auditory event are faster than responses to one of the components alone, a phenomenon known as intersensory facilitation (Hershenson, 1962; Todd, 1912). Bernstein, Rose, and Ashe (1970) varied foreperiod duration and foreperiod variability to manipulate the subject's readiness to respond at the time of presentation of the visual test stimulus. They found a larger effect of an irrelevant accessory auditory stimulus on response speed at lower levels of response readiness. Because response speed is likely to benefit more from an increase in response readiness when the initial level of readiness is low, this result suggests that intersensory facilitation operates at least in part by enhancing response readiness. Reviewing the evidence, Nickerson (1973) concluded that readiness enhancement by itself provides a sufficient $e x-$ planation for intersensory facilitation of reaction time.

Intersensory facilitation is not restricted to simple reaction time tasks or to simultaneous presentation of the test stimulus and accessory stimulus. Bernstein, Clark, and 
Edelstein (1969) found reaction time benefits in a visual choice reaction time task when the accessory tone preceded the response by about $200 \mathrm{msec}$ or more, and similar findings have been reported by Posner, Nissen, and Klein (1976).

These findings motivate the central hypothesis pursued in this paper. It seems possible that the response signal may serve a dual role, not only acting as a response signal proper that initiates guessing processes but also enhancing the speed of regular responses and thus causing a violation of the signal-independence aspect of the temporalindependence assumption of the decomposition technique. As will be discussed next, such a violation would cause the decomposition technique to underestimate or overestimate parameters of the guessing processes in systematic ways.

The speed-accuracy decomposition technique provides an estimate of the distribution of guessing durations from the comparison of the distributions of reaction times on regular trials with those on signal trials (for details, see Meyer et al., 1988). Logically, the latter two distributions will be identical up to the time of onset of the response signal. For later responses, the reaction time distribution for signal trials will be compressed relative to the distribution for regular trials. According to the present hypothesis, two factors contribute to this compression. First, relatively slow regular processes will lose the race against guessing processes on signal trials. Second, the speed of regular responses will be enhanced by the response signal. In the speed-accuracy decomposition technique, the difference between the reaction time distributions for regular and signal trials is attributed solely to the first factor, the race between regular and guessing processes. Consequently, on signal trials on which regular processes would normally lose the race against the guessing processes, but now, with the facilitation due to the response signal, win and cause a regular response, the decomposition technique will tend to classify that response incorrectly as a guess. I will refer to such incorrectly classified regular responses as spurious guesses.

The presence of spurious guesses will have two effects on the accuracy of the estimation of the parameters of the guessing processes by means of the decomposition technique. First, because spurious guesses are actually regular and highly accurate responses, the decomposition technique will tend to overestimate the accuracy of guesses. Second, because for spurious guesses the guessing processes are incorrectly declared winners of the race against regular processes, such guesses will appear to be especially fast, causing the decomposition technique to underestimate the average duration of guessing processes.

It is important to note that the degree to which estimated values for mean duration and accuracy of guess responses, as estimated by the decomposition technique, will deviate from the actual values, depends directly on the number of spurious guesses relative to the total estimated number of guesses. Appendix A demonstrates that, given two simplifying assumptions, the relative number of spurious guesses will increase with response-signal lag whenever the hazard function of the reaction time distribution for the primary task is strictly increasing. Both normal distributions and convolutions of normal and exponential distributions (exnormal distribution) have strictly increasing hazard functions (Luce, 1986). Because empirical reaction time distributions have commonly been found to be fitted very well by exnormal distributions (Ratcliff, 1979), it seems possible that facilitating effects of the response signal on regular responses may have been responsible, at least in part, for the observed effects of signal lag on the estimated guessing accuracy and mean guessing duration.

In this paper, I will adopt a model for performance in response-signal paradigms that I call the pure-guess model. According to the pure-guess model, guessing processes do not have access to preliminary, partial output of regular processes; consequently, guesses will be at chance accuracy. The adoption of this model leads to the strongest possible version of the hypothesis presented above, in which it is assumed that the above-chance guessing accuracies, as produced by the decomposition technique, can be explained entirely in terms of facilitating effects of the response signal on regular responses. The first step in validating the pure-guess model will be to look for evidence that such facilitating effects are actually present; this is the purpose of Experiment 1.

\section{EXPERIMENT 1}

Unfortunately, possible facilitating effects of response signals on regular responses cannot be demonstrated in any direct way. This is because regular responses cannot be extracted individually from the mixture of regular and guess responses on signal trials. But it is possible to pursue an indirect approach to obtain evidence concerning the existence and nature of such facilitating effects, by means of the following argument. Effects of an irrelevant accessory stimulus on the speed of regular responses are commonly explained by assuming that the accessory stimulus produces an automatic enhancement in subjects' alertness and readiness to respond (Nickerson, 1973; Posner et al., 1976). It seems reasonable to assume that a response signal, which calls for an immediate response, would exert a stronger facilitating effect on regular processes than would a completely irrelevant accessory stimulus. Therefore, if for any given task a facilitating effect of irrelevant accessory stimuli can be demonstrated, this result would support the notion that response signals also enhance the speed of regular responses when the responsesignal method is applied to the same task.

In Experiment 1, I applied this logic to the dual-string lexical decision task that has been studied extensively with the speed-accuracy decomposition technique (Meyer et al., 1988). In this task, the test stimuli consist of two letter strings, simultaneously presented one above the other. Four different combinations are possible: Both strings can be words (WW), one string can be a word and 
the other a nonword (WN or NW), or both strings can be nonwords (NN). The subjects have to respond "same" if the lexical status of the two strings is the same (WW or NN) and "different" if the lexical status of the two strings is different (WN or NW). An irrelevant auditory tone is presented at some time after the onset of the test stimulus on a random $50 \%$ of the trials.

\section{Method}

Subjects. Six subjects (4 male; age range, 18-23) participated in return for payment. They were native speakers of English and had normal or corrected-to-normal vision and hearing. Each subject participated in two experimental sessions of about $2 \mathrm{~h}$. The subjects were paid \$3 per hour plus a bonus based on performance.

Apparatus. Visual test stimuli were presented in a dimly lit room on a DEC VT-11 CRT display at a viewing distance of about $1 \mathrm{~m}$ from the subject. The accessory stimulus was a tone $(1500 \mathrm{~Hz}, 10$ msec duration, $65-\mathrm{dB}$ amplitude), generated by a Schlumberger sinesquare audio generator (Model SG-18A) and presented over a speaker located directly above the display.

The subjects responded by squeezing one of two zero-displacement dynamometers (Daytronic linear velocity force transducers, Model 152A, with conditioner amplifiers, Model 830A) with the left or right hand, as a function of the test stimulus. The system generated a voltage proportional to the force applied to the transducer, giving a continuous recording of the force output of both hands. A Schmitt trigger could be set to any preselected force level, so that when the exerted force reached this level, the system recorded the occurrence of an overt criterion response. At the beginning of the first session, each subject's maximum force level was determined for each hand separately. Criterion values were then set at $10 \%$ of the maximum force leveis. During the first two blocks in the first session, the subject heard a click presented over the speaker when the response force exceeded the criterion.

Stimuli. The test stimuli were 200 common four-letter words. In each of these words, one of the letters was replaced so that an orthographically regular, pronounceable nonword would result; letters at the four positions were replaced equally often.

The words and nonwords were used to create four types of test stimuli: WW, WN, NW, and NN pairs. The WW and NN stimuli required a positive ("same") response, whereas the WN and NW stimuli required a negative ("different") response. The four test stimulus types occurred equally often and in randomized order. The assignment of words and nonwords to the stimulus types was randomized with the restrictions that a pair could not consist of identical letter strings or a word and its corresponding nonword.

The letter strings appeared in uppercase letters in the middle of the display screen, with one string directly below the other. Each letter subtended $0.3^{\circ}$ of visual angle in width and $0.4^{\circ}$ in height. The whole stimulus subtended visual angles of about $1.4^{\circ}$ horizontally and $1.0^{\circ}$ vertically.

In the second session, the accessory tone was presented on a random $50 \%$ of the trials, some time after the onset of the visual stimulus. New randomizations were prepared for each subject and each session.

Design. The two sessions were conducted on separate days, with no more than 2 days between the sessions. The first session, which served as practice, included 12 blocks with 80 trials per block. The second session included 16 blocks, the first two of which served as training and were excluded from analysis.

Procedure. At the start of each trial, a fixation dot appeared in the middle of the screen. After $\mathbf{5 0 0}$ msec, the test stimulus appeared, with one string above and the other below the fixation dot; it remained on for $2,000 \mathrm{msec}$, after which the screen was blank for a 1,500 -msec intertrial interval.

The subjects were instructed to make a positive response if the two letter strings had the same lexical status, and a negative response otherwise; the assignment of positive and negative responses to response hands was balanced over subjects. The subjects were instructed to respond as quickly as possible before the test stimulus expired, while maintaining a high level of accuracy. Prior to the second session, the subjects were informed that a tone would be presented on a random half of the trials. They were told that the tones were irrelevant to the task that they had to perform, and they were asked to ignore the tones as much as possible. After an error or a late response (over $2,000 \mathrm{msec}$ ), the message "Error or Too Late" appeared on the screen, remaining there until the subject initiated the next trial by squeezing one of the dynamometers.

A bonus system was used to encourage the subjects to follow the instructions. The subjects earned 0.50 cent for a correct response within $1,000 \mathrm{msec}$ and 0.25 cent for a correct response between 1,000 and $2,000 \mathrm{msec}$; they lost 3 cents for every incorrect or late response. After each block, the bonus earned during that block was displayed, together with the total bonus earned so far in the session. The average bonus earned by subjects was about 25 cents per block of trials.

Signal lags. The lags for the accessory stimulus in the second session were kept fixed throughout the session. Separate lags were used for each subject and for each type of test stimulus. The computation of these lags was based on the subject's performance during the last six blocks of the first session. The subtraction of $100 \mathrm{msec}$ from the 10 th percentile point of the reaction time distribution obtained with each type of test stimulus during those blocks yielded the signal lags for the second session. The average signal lags in milliseconds for each stimulus type were 626 (range: 452-739) for WW, 757 (range: 624-912) for NN, 692 (range: 570-825) for WN, and 707 (range: 556-830) for NW stimuli.

\section{Results}

The data for 1 subject were excluded from analysis because his performance had improved so much by the second session that almost all responses preceded the onset of the accessory stimulus.

Mean reaction times, averaged across subjects, are presented in Table 1, separately for each stimulus type and for regular and signal trials. Replicating previous findings (e.g., those of Meyer et al., 1988), positive responses were considerably faster for WW stimuli than for NN stimuli, whereas negative responses for WN and NW stimuli had intermediate reaction times $[F(3,12)=10.4$, $p<.01]$. Reaction times were faster for signal trials than for regular trials; the overall effect of trial type was 20 msec $[F(1,4)=11.5, p<.03]$. The interaction of stimulus type and trial type was not significant $(F<1)$.

Mean overall response accuracies are presented in Table 1, separately for each stimulus type and trial type. There were no significant effects on accuracy.

The accessory stimulus effect in this experiment is similar in size to the effects found in other studies (Bernstein

Table 1

Experiment 1: Results for Regular and Signal Trials

\begin{tabular}{lrrrrr}
\hline & \multicolumn{2}{c}{$\begin{array}{c}\text { Mean RT } \\
\text { (in msec) }\end{array}$} & & \multicolumn{2}{c}{$\begin{array}{c}\text { Response Accuracy } \\
\text { (\% Correct) }\end{array}$} \\
\cline { 2 - 3 } \cline { 6 - 7 } \multicolumn{1}{c}{ Stimulus Type } & Regular & Signal & & Regular & Signal \\
\hline Word-word & 932 & 909 & & 94.1 & 93.3 \\
Nonword-nonword & 1,093 & 1,080 & & 96.5 & 96.8 \\
Word-nonword & 1,001 & 975 & & 95.8 & 94.9 \\
Nonword-word & 990 & 973 & & 96.1 & 96.7 \\
\hline
\end{tabular}


et al., 1969; Posner et al., 1976). For the purposes of the present paper, it will be useful to assess the effect of the accessory stimulus on the speed of regular processes in more detail. The main hypothesis pursued in this paper holds that a response signal may serve to enhance the speed of regular processes, so that on some trials these processes will win the race against guessing processes that they otherwise would have lost. From the data presented by Meyer et al. (1988), guessing durations tend to range from 150 to $300 \mathrm{msec}$. It is therefore especially important to assess the effects of the accessory stimulus on regular responses that occur in the range from about 150 to about 300 msec after onset of the accessory stimulus.

To estimate the magnitude of the accessory stimulus effect at several different points since onset of the accessory stimulus, four intervals of $150-, 200-, 250-$, and $300-$ msec duration were defined, each interval starting at the onset time of the accessory stimulus. The number of responses falling within each of these intervals was determined, separately for the four types of test stimulus and for regular and signal trials. These numbers, expressed as the proportion of the total number of responses occurring after the onset of the accessory stimulus and averaged across subjects, are presented in Table 2 . Overall, relatively more responses occurred within the intervals on signal trials than on regular trials $[F(1,4)=15.0$, $p<.02]$. However, this effect was not obtained for the shortest interval (150 msec), as is evidenced by a significant interaction of interval length and trial type $[F(3,12)$ $=8.23, p<.01]$. The effect appears to have been somewhat larger for NW stimuli than for the other stimulus types, but the interaction of stimulus type and trial type did not approach significance $[F(3,12)=1.82, p>.15]$. There were no differences between regular and signal trials with regard to the proportion incorrect responses within each of the intervals.
These results were used to estimate the size of the accessory effect at various times since onset of the accessory stimulus. For regular trials, I determined the lengths of the intervals that contained the same proportions of responses as were found for signal trials with the original intervals. The difference between the original and the corresponding intervals provides an estimate (in milliseconds) of the size of the accessory effect at the end of each corresponding interval. Conceptually, this estimate indicates, for various percentile points, by how many milliseconds the cumulative reaction time distribution function for regular trials must be shifted in the direction of the onset of the accessory stimulus, so that it will overlap the corresponding percentile points in the distribution for signal trials. The resulting values, averaged across subjects and separately for each type of test stimulus, are presented in Table 2. The size of the accessory effect increased monotonically as a function of interval length from about $\mathbf{0 ~ m s e c}$ for the shortest to about $\mathbf{4 0 ~} \mathrm{msec}$ for the longest interval $[F(3,12)=7.67, p<.01]$. None of the other effects approached significance.

An estimate for the relative strength of the accessory effect at different time points can be obtained by expressing the size of the effect as a proportion of the time since onset of the accessory stimulus. The resulting proportionality factors, referred to as facilitation factors, are presented in Table $2 .{ }^{2}$ The facilitation factor exhibited a rapid increase from about .0 at the shortest time $(150 \mathrm{msec})$ to about .12 at the longer times.

\section{Discussion}

The results of Experiment 1 indicate that accessory stimuli have a consistent effect on the speed of responses in the dual-string lexical decision task. Detailed analysis indicated that responses that occurred within about $150 \mathrm{msec}$ from the onset of the accessory stimulus re-

Table 2

Experiment 1: Accessory Effects as Functions of Time Since Onset of the Accessory Stimulus

\begin{tabular}{|c|c|c|c|c|c|}
\hline \multirow[b]{2}{*}{ Stimulus Type } & \multirow[b]{2}{*}{ Interval } & \multicolumn{2}{|c|}{ \% Responses } & \multirow{2}{*}{$\begin{array}{c}\text { Accessory } \\
\text { Effect } \\
\text { (in msec) }\end{array}$} & \multirow{2}{*}{$\begin{array}{c}\text { Facilitation } \\
\text { Factor }\end{array}$} \\
\hline & & Regular & Signal & & \\
\hline Word-word & $\begin{array}{l}150 \\
200 \\
250 \\
300\end{array}$ & $\begin{array}{l}33.8 \\
43.2 \\
53.4 \\
65.6\end{array}$ & $\begin{array}{l}34.2 \\
46.8 \\
61.4 \\
72.0\end{array}$ & $\begin{array}{r}3 \\
17 \\
31 \\
37\end{array}$ & $\begin{array}{l}.02 \\
.08 \\
.11 \\
.11\end{array}$ \\
\hline Nonword-nonword & $\begin{array}{l}150 \\
200 \\
250 \\
300\end{array}$ & $\begin{array}{l}30.0 \\
38.6 \\
45.6 \\
54.0\end{array}$ & $\begin{array}{l}31.6 \\
42.2 \\
52.8 \\
62.6\end{array}$ & $\begin{array}{r}-1 \\
18 \\
32 \\
41\end{array}$ & $\begin{array}{r}-.01 \\
.08 \\
.11 \\
.12\end{array}$ \\
\hline Word-nonword & $\begin{array}{l}150 \\
200 \\
250 \\
300\end{array}$ & $\begin{array}{l}35.0 \\
43.8 \\
52.6 \\
61.4\end{array}$ & $\begin{array}{l}33.8 \\
46.4 \\
57.6 \\
67.4\end{array}$ & $\begin{array}{r}-7 \\
21 \\
34 \\
34\end{array}$ & $\begin{array}{r}-.05 \\
.09 \\
.12 \\
.10\end{array}$ \\
\hline Nonword-word & $\begin{array}{l}150 \\
200 \\
250 \\
300\end{array}$ & $\begin{array}{l}33.0 \\
42.2 \\
51.0 \\
60.0\end{array}$ & $\begin{array}{l}36.0 \\
51.4 \\
61.8 \\
70.0\end{array}$ & $\begin{array}{r}5 \\
29 \\
47 \\
49\end{array}$ & $\begin{array}{l}.03 \\
.13 \\
.16 \\
.14\end{array}$ \\
\hline
\end{tabular}


mained unaffected, whereas responses that occurred about 200 msec or more after its onset were speeded up considerably by the accessory stimulus. The "dead time" of about $150 \mathrm{msec}$ for facilitation to take effect undoubtedly reflects in part the time required for the processing of the response signal. In addition, it is possible that response execution processes that immediately precede the criterion response may have remained relatively unaffected by the accessory stimulus.

It should be noted that different signal lags were used for the different stimulus types. This was done to ensure functionally similar signal lags for the different stimulus types, but it also resulted in a confounding between stimulus type and signal lag. This suggests the possibility that the faster responses on signal trials may have been due not to alerting effects of the accessory stimulus on regular responses but to the use of the perceived length of the signal lag as an informative cue to help in selection of the correct response. However, this possibility seems unlikely for two reasons. First, the subjects were never informed about the relation between stimulus type and signal lag, and none reported having noticed such a relation. Second, available evidence suggests that people are poor in discriminating between temporal periods of only slightly different durations like those used in the present experiment, even when such temporal discriminations constitute the primary task (e.g., as in Niemi \& Naatanen, 1981).

In conclusion, the results of this experiment support the notion that response signals may enhance the speed of regular responses. It should be emphasized that the facilitating effects obtained in Experiment 1 were due to an irrelevant accessory stimulus and that they cannot be used in any direct way to estimate the size of such effects when a response signal, which requires an immediate response, is used. However, as argued before, it seems reasonable to assume that the facilitating effects of response signals may be stronger than those obtained in the present experiment.

\section{SIMULATIONS}

The results of Experiment 1 supported the central assumption of the pure-guess model-that the response signal serves to speed up regular responses, resulting in a violation of the signal-independence assumption. Earlier, I showed qualitatively how this model can account for the observed effects of signal lag on estimated guessing accuracy and mean guessing duration in the decomposition technique. In this section, I will report the results from two Monte Carlo simulation studies in which the pureguess model was tested quantitatively against two representative sets of results from the speed-accuracy decomposition technique (Meyer et al.,1988, Experiments 3 and 5). The simulations involved violations of both the signal-independence and the stochastic-independence aspects of the temporal-independence assumption. Thus, presentation of the response signal was assumed to en- hance the speed of regular responses, and the durations of regular and guessing processes on signal trials were allowed to covary on a trial-by-trial basis.

The simulation procedure was as follows. Distribution functions of regular-trial responses and of guessing durations were obtained by fitting a convolution of a nor$\mathrm{mal}$ and an exponential distribution to the respective empirical cumulative distribution functions reported by Meyer et al. (1988). As has commonly been observed (Ratcliff, 1979), the resulting functions provided an excellent fit to the empirical ones. A moderate speed-accuracy tradeoff was assumed for regular responses, to incorporate the fact that errors were found to be somewhat faster than correct responses in the two data sets under consideration. $^{3}$

On each trial in the simulations, samples were drawn pseudorandomly from these distributions, resulting in a specific correlation between the durations of regular and guessing responses. ${ }^{4}$ One of the response-signal lags used in the simulation was added to the sampled guessing duration to obtain the guessing-completion time for that trial.

The effect of the response signal on the speed of regular responses was implemented as follows. As is suggested by the results of Experiment 1, the relative strength of the facilitating effect, represented by the facilitation factor, increases as a function of time since signal onset, up to an asymptotic value. In the present simulations, the facilitation factor was assumed to rise linearly from zero at the time of signal onset to a maximum value at $300 \mathrm{msec}$ after signal onset; for longer times, the facilitation factor remained constant at the maximum value. The response times for regular responses that occurred after onset of the response signal were modified as follows: The interval between the original response time and the time of signal onset was computed, the resulting value was multiplied by the facilitation factor for that value, and the product was subtracted from the original response time to give the modified regular response time. As an example, suppose that the maximum facilitation factor is 0.3 , and consider regular responses occurring at $0,100,200$, 300 , and $400 \mathrm{msec}$ after response-signal onset. The facilitation factors for these responses are $0.0,0.1,0.2,0.3$, and 0.3 , respectively, resulting in modified response times of $0,90,160,210$, and $280 \mathrm{msec}$ after signal onset.

To implement the race model assumptions, the actual response time on each trial was set to the modified regular response time or to the guessing-completion time, whichever was smaller. If the regular response was faster, the accuracy of the response was set equal to the accuracy of the original response. Possible effects of facilitation on the accuracy of regular responses were not taken into account because, as the results of Experiment 1 suggest, such effects are likely to be small and to ignore them would not significantly affect the simulation results. If the guess response won, its accuracy was set to $50 \%$, as the pure-guess model required.

The simulations involved 20,000 trials for each signal lag used. Subsequently, the results of the simulated ex- 
periment were subjected to the speed-accuracy decomposition analysis described by Meyer et al. (1988), to estimate the distribution of guessing durations and the average accuracy of guess responses at each signal lag. ${ }^{5}$

The simulations involved two free parameters, the maximum facilitation factor and the correlation between regular and guessing responses. Best-fitting parameters were obtained by minimizing the sum of the squared differences between simulated and empirical guessing accuracies for the various response signal lags, using a minimization routine (STEPIT). This procedure provides a strong test of the pure-guess model, because the model must account not only for changes in estimated guessing accuracy as a function of signal lag but also for the effects of signal lag on the mean reaction times for correct and incorrect responses and on the estimated mean guessing durations.

Chi-square tests were used to evaluate the goodness of fit. The two free parameters of the model were taken into account when the fit for accuracy-related variables was evaluated; for other, time-related variables, the fit was considered to be parameter-free.

\section{Simulation 1}

In the first simulation, the pure-guess model was fitted to the results for Experiment 3 in Meyer et al. (1988), who employed the dual-string lexical decision task described earlier. Using one short signal lag and several longer ones, Meyer et al. found that guessing accuracy rose from about $50 \%$ for the shortest to about $70 \%$ for the next shortest lag, after which guessing accuracy seemed to have reached a plateau and did not increase further for still longer signal lags. A possible interpretation of this result is that it reflects a discrete partial output of the processing system before it has reached a decision (Meyer et al.). However, as pointed out by Ratcliff (1988), there was a gap of about $275 \mathrm{msec}$ between the shortest and the next shortest signal lag used, so that it is not known whether during that interval there was a gradual increase in guessing accuracy or a discrete jump. To address this issue, the response signal lags used in the simulation were set to the average lags used in the origi-

Table 3

Simulation 1: Results For the Regular and Signal Trials

\begin{tabular}{|c|c|c|c|c|c|c|c|}
\hline \multirow{3}{*}{$\begin{array}{l}\text { Trial } \\
\text { Type }\end{array}$} & \multirow{3}{*}{$\begin{array}{l}\text { Signal Lag } \\
\text { (in msec) }\end{array}$} & \multicolumn{4}{|c|}{$\begin{array}{l}\text { Mean RT } \\
\text { (in msec) }\end{array}$} & \multirow{2}{*}{\multicolumn{2}{|c|}{$\begin{array}{l}\text { Response } \\
\text { Accuracy } \\
\text { (\% Correct) }\end{array}$}} \\
\hline & & \multicolumn{2}{|c|}{$\begin{array}{c}\text { Correct } \\
\text { Responses }\end{array}$} & \multicolumn{2}{|c|}{$\begin{array}{c}\text { Incorrect } \\
\text { Responses }\end{array}$} & & \\
\hline & & Emp & Sim & Emp & Sim & $\overline{\text { Emp }}$ & $\overline{\text { Sim }}$ \\
\hline Regular & & 767 & 767 & 719 & 720 & 92.7 & 92.7 \\
\hline Signal & 150 & 411 & 388 & 384 & 384 & 52.9 & 53.6 \\
\hline Signal & 250 & & 476 & & 476 & & 59.0 \\
\hline Signal & 350 & & 555 & & 563 & & 66.5 \\
\hline Signal & 425 & 612 & 612 & 621 & 627 & 73.1 & 72.0 \\
\hline Signal & 475 & 648 & 649 & 664 & 672 & 76.9 & 75.5 \\
\hline Signal & 525 & 684 & 680 & 713 & 713 & 79.4 & 77.9 \\
\hline Signal & 575 & 715 & 706 & 749 & 757 & 81.6 & 80.3 \\
\hline
\end{tabular}

Note-Emp $=$ empirical results. $\mathrm{Sim}=$ simulation results.
Table 4

Simulation 1: Results Regarding the Guessing Processes on Signal Trials

\begin{tabular}{|c|c|c|c|c|c|c|}
\hline \multirow{2}{*}{$\begin{array}{l}\text { Signal Lag } \\
\text { (in msec) }\end{array}$} & \multicolumn{2}{|c|}{$\begin{array}{l}\text { Duration } \\
\text { (in msec) } \\
\end{array}$} & \multicolumn{2}{|c|}{$\begin{array}{c}\text { Interquartile } \\
\text { Range } \\
\text { (in msec) } \\
\end{array}$} & \multicolumn{2}{|c|}{$\begin{array}{c}\text { Response } \\
\text { Accuracy } \\
\text { (\% Correct) } \\
\end{array}$} \\
\hline & Emp & Sim & Emp & Sim & Emp & Sim \\
\hline $\begin{array}{l}150 \\
250 \\
350\end{array}$ & 239 & $\begin{array}{l}237 \\
227 \\
212\end{array}$ & 65 & $\begin{array}{l}64 \\
60 \\
58\end{array}$ & 52.9 & $\begin{array}{l}53.5 \\
58.4 \\
64.3\end{array}$ \\
\hline 425 & 201 & 200 & 60 & 62 & 68.2 & 67.6 \\
\hline 475 & 198 & 197 & 66 & 63 & 69.7 & 68.3 \\
\hline 525 & 200 & 193 & 65 & 64 & 68.4 & 68.9 \\
\hline 575 & 206 & 190 & 68 & 66 & 68.8 & 69.3 \\
\hline
\end{tabular}

Note-Emp $=$ empirical results. Sim $=$ simulation results.

nal experiment ( $150,425,475,525$, and $575 \mathrm{msec})$, but two intermediate lags (250 and $350 \mathrm{msec}$ ) were added.

The parameters of the convolution of normal and exponential distributions were obtained by fitting the empirical cumulative distribution functions for regular-trial reaction times, averaged, by means of Vincent (horizontal) averaging, across stimulus type (Meyer et al., 1988, Figure 15). ${ }^{6}$ The resulting parameters were $590 \mathrm{msec}$ for the mean and $81 \mathrm{msec}$ for the standard deviation of the normal, and $232 \mathrm{msec}$ for the time constant of the exponential. The corresponding parameters used in fitting the empirical guessing-duration distribution function were 199,46 , and $40 \mathrm{msec}$; only the guessing-duration distribution function for the shortest signal lag (150 msec) was used to estimate these parameters, because only the function for this lag would be relatively uncontaminated by spurious guesses. The parameters of the pure-guess model that provided the best fit to the empirical guessing accuracies were 0.31 for the maximum facilitation factor and -0.40 for the trial-by-trial correlation between the durations of regular and guessing processes.

The simulation results for the median latencies for correct and incorrect responses and for the overall accuracy for regular and signal trials are presented in Table 3. The corresponding empirical values, calculated by averaging the results reported by Meyer et al. (1988, Table 8) across stimulus type, are also presented in this table. The fit is fairly good for each of the variables $\left[\chi^{2}(5)=1.50\right.$, $p>.91$, for correct response latency; $\chi^{2}(5)=.24$, $p>.999$, for incorrect response latency; $\chi^{2}(3)=0.10$, $p>.99$, for overall response accuracy].

Simulation results and empirical results for the median and the interquartile range of the estimated guessing duration distributions and for the estimated guessing accuracies are presented in Table 4. The empirical values were calculated by averaging the results reported by Meyer et al. (1988, Table 9) across stimulus types. The model provided fairly good fits for each of the guessing-related variables $\left[\chi^{2}(5)=1.63, p>.89\right.$, for median guessing duration; $\chi^{2}(5)=0.30, p>.99$, for interquartile range; $\chi^{2}(3)=0.06, p>.99$, for guessing accuracy].

The distribution function for regular-trial response times that was used in the simulation is shown in Figure 1; also 


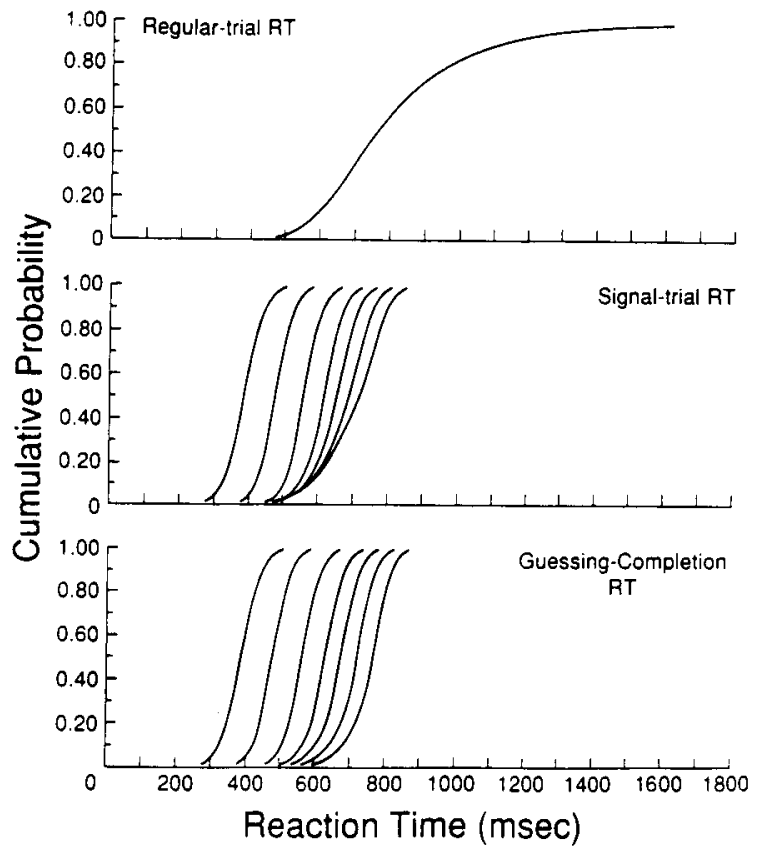

Figure 1. Cumulative distribution functions in Simulation 1. The top, middle, and bottom panels illustrate, respectively, the reaction times (RTs) on regular trials, the reaction times on signal trials, and the derived guessing-completion times.

shown in this figure are the distribution functions produced by the simulation for response times on signal trials and for guessing-completion times at each signal lag. These functions can be compared to the empirical functions reported by Meyer et al. (1988, Figure 15). The simulated and empirical distribution functions can be seen to correspond quite well. In particular, the simulated and the empirical functions for signal-trial reaction times show essentially the same horizontal shifts with increasing signal lag, as well as the same changes in steepness. Also, both the simulated and the empirical functions for guessingcompletion times for the different lags are essentially parallel, with some minor deviations in the lower tails at the longer signal lags.

The results of this simulation demonstrate the potential of the pure-guess model to account for the results from the speed-accuracy decomposition technique, results that have been considered conclusive evidence that guesses are based on partial processing output (Meyer et al., 1988; Ratcliff, 1988). There is another interesting conclusion that may be drawn from the simulation results. The results for the intermediate signal lags (250 and $350 \mathrm{msec}$ ) suggest that the presence of a guessing accuracy plateau at longer signal lags, as observed by Meyer et al. (1988) and as obtained in the present simulation, does not necessarily imply that such a plateau was reached in a discrete jump, as when the plateau is assumed to reflect a discrete intermediate processing output. Rather, such a plateau appears to represent an asymptotic level to which estimated guessing accuracy rises gradually.
If one assumes temporal independence between regular and guessing processes, it is possible to estimate not only the overall accuracy of guess responses for a particular signal lag but also the speed-accuracy microtradeoff for the guess responses. The derivation of this microtradeoff is discussed in Appendix B. For each of the signal lags in the present simulation, the distribution of guesscompletion times was subdivided into deciles, and the average guessing accuracy was computed for each of the deciles. In Figure 2, the resulting guessing accuracies are plotted against the average guess-completion time for each decile, separately for each signal lag. The estimated microtradeoff can be seen to change in an interesting way as a function of signal lag. For short lags, the tradeoff is positive, because slower guess responses are more accurate; but the tradeoff becomes distinctly negative for longer lags, with faster guess responses being more accurate than slower ones.

An intuitive understanding of the changes in the estimated guess response microtradeoff with signal lag can be obtained in the following way. At short signal lags, regular processes do not have a significant chance to win the race against guessing processes unless the latter processes are relatively slow. Hence, spurious guesses will be rare among relatively fast guess responses but become more prevalent in the slower range of guess responses, causing an increase of estimated guessing accuracy as guess response time increases. At long lags, on the other hand, spurious guesses will contribute to the entire range of guess response times. However, because, as explained before, spurious guesses will appear to be especially fast guesses, they will be represented especially well among the faster guess response times. In fact, for long signal lags, the fastest estimated guess response times will be due almost exclusively to spurious guesses. This explains why, at the longer signal lags, estimated guessing accuracy for the fastest guess responses approaches

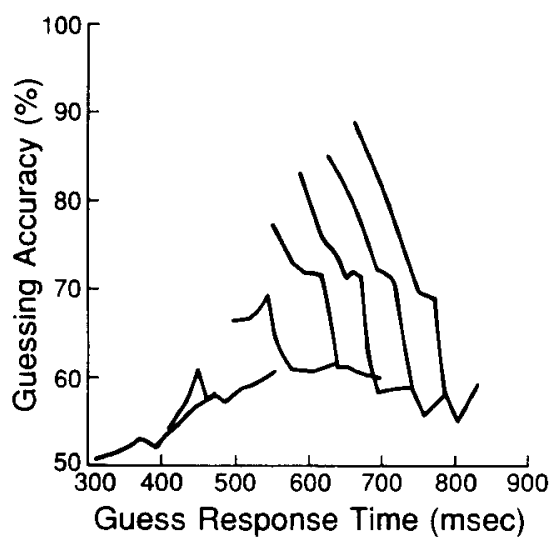

Figure 2. Speed-accuracy microtradeoffs for guess responses in Simulation 1. Tradeoffs for guess responses at each of the response signal lags are shown, arranged from lower left to upper right in order of ascending signal lag. 
the accuracy of regular responses and gradually decreases for slower guess responses.

Unfortunately, empirically estimated speed-accuracy microtradeoffs for guess responses are presently not available for comparison to the simulated ones. The procedure for estimating the microtradeoffs and the simulation results are described here for future reference, because these tradeoffs provide a powerful means of distinguishing between the pure-guess model and the sophisticated-guessing model. This point will be discussed in detail in Experiment 2 .

\section{Simulation 2}

In the second simulation, the pure-guess model was fitted to the results of an experiment by Meyer et al. (1988, Experiment 5) in which a single-string lexical decision task was used. The speed-accuracy decomposition technique gave quite different results for this task than for the previously discussed dual-string lexical decision taskso different, in fact, that Meyer et al. concluded that they constituted two qualitatively different patterns. Guessing accuracy was found to increase gradually as a function of signal lag, reaching at least $80 \%$, and there were clear effects of signal lag on the mean and the interquartile range of the guessing duration distribution. Meyer et al. argued that such a pattern of results would be more characteristic of a continuous accumulation of information during input processing, whereas the pattern of results obtained in the dual-string lexical decision task would be more characteristic of a discrete intermediate processing state. The ability to account for these two different patterns of results by means of the same mechanism would provide compelling support for the pure-guess model.

In the simulation, the signal lags were the same as those in the original experiment, ranging from 100 to $275 \mathrm{msec}$ in steps of $35 \mathrm{msec}$. The parameters of the convolution of normal and exponential distributions used in fitting the empirical regular reaction time distribution, averaged across stimulus type (word-nonword, Meyer et al., 1988, Figure 20), were $395 \mathrm{msec}$ for the mean and $50 \mathrm{msec}$ for the standard deviation of the normal, and $73 \mathrm{msec}$ for the time constant of the exponential. Guessing accuracy for

Table 5

Simulation 2: Results for the Regular and Signal Trials

\begin{tabular}{|c|c|c|c|c|c|c|c|}
\hline \multirow{3}{*}{$\begin{array}{l}\text { Trial } \\
\text { Type }\end{array}$} & \multirow{3}{*}{$\begin{array}{l}\text { Signal Lag } \\
\text { (in msec) }\end{array}$} & \multicolumn{4}{|c|}{$\begin{array}{l}\text { Mean RT } \\
\text { (in msec) }\end{array}$} & \multirow{2}{*}{\multicolumn{2}{|c|}{$\begin{array}{c}\text { Response } \\
\text { Accuracy } \\
\text { (\% Correct) }\end{array}$}} \\
\hline & & \multicolumn{2}{|c|}{$\begin{array}{c}\text { Correct } \\
\text { Responses }\end{array}$} & \multicolumn{2}{|c|}{$\begin{array}{c}\text { Incorrect } \\
\text { Responses }\end{array}$} & & \\
\hline & & Emp & Sim & Emp & Sim & Emp & Sim \\
\hline Regular & & 455 & 454 & 435 & 436 & 94.9 & 94.9 \\
\hline Signal & 100 & 324 & 325 & 316 & 317 & 62.7 & 62.7 \\
\hline Signal & 135 & 344 & 349 & 336 & 347 & 66.6 & 68.0 \\
\hline Signal & 170 & 364 & 368 & 358 & 370 & 73.8 & 73.7 \\
\hline Signal & 205 & 385 & 385 & 379 & 394 & 78.6 & 79.2 \\
\hline Signal & 240 & 403 & 400 & 404 & 420 & 84.9 & 83.7 \\
\hline Sienal & 275 & 423 & 415 & 432 & 440 & 89.3 & 87.6 \\
\hline
\end{tabular}

Note-Emp $=$ empirical results. Sim $=$ simulation results.
Table 6

Simulation 2: Results Regarding the Guessing Processes on Signal Trials

\begin{tabular}{|c|c|c|c|c|c|c|}
\hline \multirow{2}{*}{$\begin{array}{c}\text { Signal Lag } \\
\text { (in msec) }\end{array}$} & \multicolumn{2}{|c|}{$\begin{array}{l}\text { Duration } \\
\text { (in msec) }\end{array}$} & \multicolumn{2}{|c|}{$\begin{array}{c}\text { Interquartile } \\
\text { Range } \\
\text { (in msec) } \\
\end{array}$} & \multicolumn{2}{|c|}{$\begin{array}{c}\text { Response } \\
\text { Accuracy } \\
\text { ( } \$ \text { Correct) } \\
\end{array}$} \\
\hline & Emp & $\operatorname{Sim}$ & Emp & Sim & Emp & $\mathrm{Sim}$ \\
\hline 100 & 222 & 224 & 51 & 58 & 61.1 & 60.9 \\
\hline 135 & 207 & 215 & 62 & 61 & 63.4 & 65.0 \\
\hline 170 & 198 & 205 & 64 & 65 & 69.2 & 69.5 \\
\hline 205 & 187 & 195 & 64 & 71 & 73.1 & 73.6 \\
\hline 240 & 186 & 185 & 79 & 77 & 78.0 & 77.9 \\
\hline 275 & 190 & 179 & 93 & 86 & 82.8 & 81.9 \\
\hline
\end{tabular}

Note-Emp $=$ empirical results. Sim $=$ simulation results.

even the shortest lag used in the experiment was found to be already well above chance. According to the pureguess model, this indicates the presence of a substantial number of spurious guesses that may have seriously distorted the estimation of the distribution of guessing durations. For this reason, the parameters for the guessingduration distribution could not be estimated separately for this experiment, and the distribution used in Simulation 1 was also used in the present simulation. The parameters that provided the best fit to the empirical guessing accuracies were 0.21 for the maximum accessory factor and -0.05 for the correlation between the durations of regular and guess responses.

The simulation results for the median latencies for correct and incorrect responses and for the overall accuracy for regular and signal trials are presented in Table 5, together with the corresponding empirical values that were calculated by averaging the results reported by Meyer et al. (1988, Table 12) across stimulus type. The fit for each of the variables is quite good $\left[\chi^{2}(6)=.29, p>.999\right.$, for correct response latency; $\chi^{2}(6)=2.06, p>.91$, for incorrect response latency; $\chi^{2}(4)=.08, p>.999$, for overall response accuracy].

Simulation results and empirical results for the median and the interquartile range of the estimated guessingduration distributions and for the estimated guessing accuracies are presented in Table 6 . The empirical values were calculated by averaging the results reported by Meyer et al. (1988, Table 13) across stimulus types. Again, the model provided a fairly good fit for each of these variables $\left[\chi^{2}(6)=1.51, p>.95\right.$, for median guessing duration; $\chi^{2}(6)=2.19, p>.90$, for interquartile range; $\chi^{2}(4)=0.06, p>.999$, for guessing accuracy].

The distribution function of regular response times that was used in the simulation is shown in Figure 3; also shown in this figure are the distribution functions produced by the simulation for response times on signal trials and for guessing completion times at each signal lag. These functions can be compared to the empirical functions reported by Meyer et al. (1988, Figure 20). As in the previous simulation, the simulated and empirical distribution functions correspond quite well. Compared to the corresponding functions in Simulation 1, presented in 


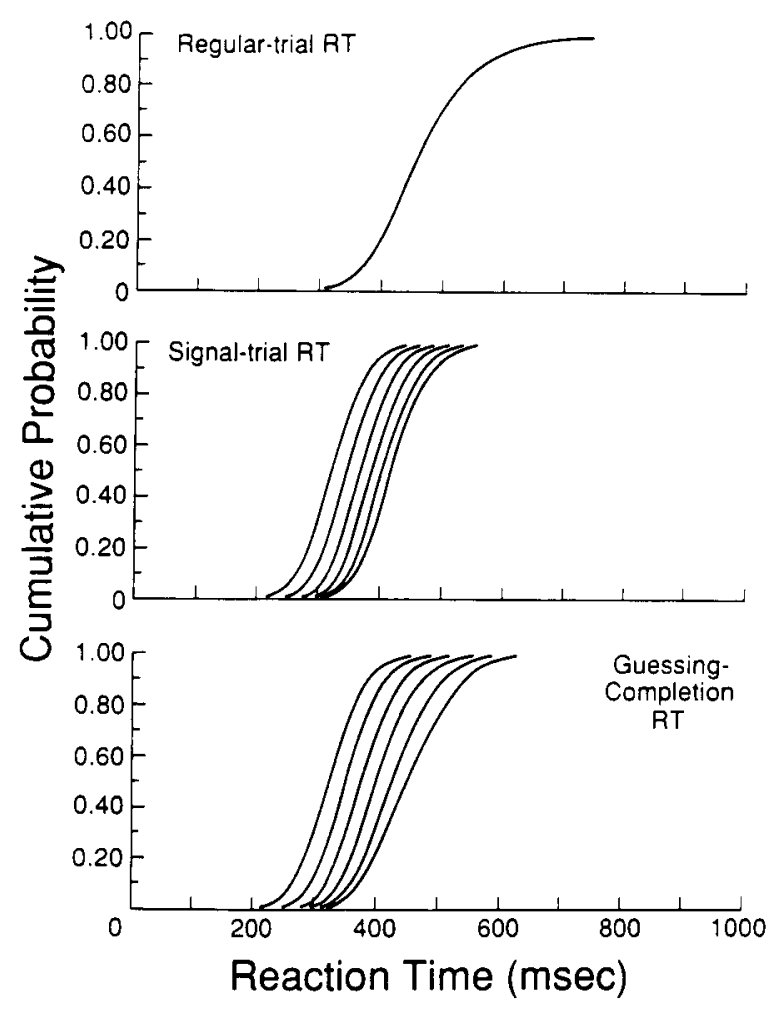

Figure 3. Cumulative distribution functions in Simulation 2. The top, middle, and bottom panels illustrate, respectively, the reaction times (RTs) on regular trials, the reaction times on signal trials, and the derived guessing-completion times.

Figure 1, the simulated distribution functions of signaltrial reaction times in the present simulation also show clear horizontal shifts with increasing signal lag, but except for the lower tails at the longest lags, these functions appear to remain essentially parallel and do not show the marked changes in steepness found in the previous simulation. This difference is also clearly present for the empirical distribution functions (Meyer et al., Figures 15 and 20). The simulated distribution functions for guessing completion times show a difference in the other direction; in the present simulation, these functions become less steep as signal lag increases, whereas in the previous simulation, the functions for the different signal lags were essentially parallel. Again, such a difference is also clearly present for the empirical functions (Meyer et al., Figures 15 and 20).

In Figure 4 are shown the estimated speed-accuracy microtradeoffs for guessing responses for each signal lag in the present simulation. The change of the microtradeoff that accompanies the changes in signal lag closely resembles the pattern found in the previous simulation (Figure 2), the tradeoff being positive at short signal lags and becoming strongly negative at the longer lags.

\section{Discussion}

The two simulation studies demonstrate that the pureguess model can fit the results from the speed-accuracy decomposition technique in considerable detail. In particular, its ability to account in essentially the same way for the quite different patterns of results for single-string and dual-string lexical decision tasks provides compelling support for the model.

In evaluating these simulation results, it is important to consider the plausibility of the values for the facilitation factor and for the trial-by-trial correlation between regular and guess responses that were used in the simulations. As expected, the best-fitting maximum facilitation factors for the response signal in both simulations were substantially larger than the ones found for the accessory stimulus in Experiment 1. Also, the finding of stronger facilitation for the dual-string than for the singlestring lexical decision task seems reasonable, since the former version of the task involves a more complex arrangement of processing operations and might therefore benefit more from facilitating effects of the response signal. Nevertheless, the large facilitation factor used in Simulation 1 represents a very substantial facilitating effect, and in the absence of direct evidence, the question of whether these effects are of a plausible magnitude cannot be completely settled at present.

The simulation procedure allowed for violations of stochastic independence of regular and guess responses, and in Simulation 1 a sizable negative correlation was used to fit the results for the dual-string lexical decision task. Such a negative correlation is perhaps best thought of as reflecting an inability to be optimally prepared at the same time for the regular task and for responding swiftly to a possible response signal (De Jong, 1991), so that efficient preparation for one task could be achieved only at the expense of less efficient preparation for, and performance of, the other task. The very small negative correlation for the single-string lexical decision task is somewhat surprising in this respect, but it seems consistent with the general finding that less complex tasks interfere less with and suffer less interference from a concurrent task (Navon \& Gopher, 1979).

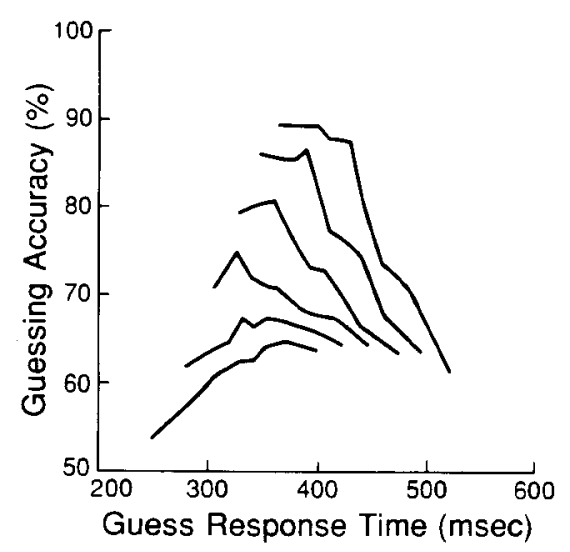

Figure 4. Speed-accuracy microtradeoffs for guess responses in Simulation 2. Tradeoffs for guess responses at each of the response signal lags are shown, arranged from lower left to upper right in order of ascending signal lag. 
The contribution of the negative correlation of regular and guess responses to the simulation results for the dualstring lexical decision task was evaluated by comparing the original results with results obtained when either the facilitation factor or the correlation was set to zero. A negative correlation alone was found to produce an only modest decrease of the mean and interquartile range of the estimated guessing-duration distributions and a slight increase of estimated guessing accuracy as signal lag increased. Comparison between the results for the different simulations suggested that the effects of facilitation and a negative correlation on the mean and interquartile range of the guessing-duration distribution are approximately additive. The effects of these factors on estimated guessing accuracy were found to combine in a more complex manner, with a negative correlation causing a significantly steeper increase of estimated guessing accuracy as signal lag increased, but not affecting the asymptotic level of accuracy.

The results from these simulation studies appear to establish the pure-guess model as a viable alternative to the sophisticated-guessing model proposed by Meyer et al. (1988). Both models appear capable of accounting for the results from the speed-accuracy decomposition technique in considerable detail. The pure-guess model has the advantage of being able to account for the different patterns of results in single- and dual-string lexical decision tasks in essentially the same way. Also, it is better capable of dealing with the opposite effects of signal lag on the mean and the interquartile range of the estimated guessingduration distribution found for the single-string lexical decision task. As discussed previously, the sophisticatedguessing model may be able to explain the effects on the mean guessing duration as foreperiod effects. However, contrary to the observed opposite effects on the mean and the interquartile range, effects of foreperiod duration on the mean and variance of simple reaction times have commonly been found to be positively correlated (for a review, see Luce, 1986). These advantages of the pure-guess model may be offset, however, by remaining concerns regarding the plausibility of the rather strong facilitating effects that the model needs to assume in order to account for the results from speed-accuracy decomposition. A more rigorous test between the two models is clearly needed; this will be the purpose of the next experiment.

\section{EXPERIMENT 2}

Two differential predictions can be derived from the pure-guess and the sophisticated-guessing models, the first concerning the issue of whether the estimated guessingduration distribution is invariant with stimulus type, and the second concerning the relation between the macro and micro speed-accuracy tradeoffs for guess responses.

The pure-guess model attributes the effects of signal lag on estimated guessing accuracy and on the guessingduration distribution to the same mechanism. Therefore, for any given signal lag, the model predicts differences between different stimulus types in both the accuracy and the distribution of guess responses if the distributions of regular responses for the different stimulus types are sufficiently different. Exploratory simulations indicated that effects of stimulus type on the guessing-duration distribution can be rather subtle, even when regular-trial response times differ substantially between stimulus types. Thus, demonstrations of possible violations of stimulustype invariance of the guessing-duration distribution may require considerable power.

The sophisticated-guessing model assumes temporal independence between regular and guessing processes and therefore predicts stimulus-type invariance of the guessing-duration distribution. In support of this prediction, no evidence for violations of stimulus-type invariance has been found in any study to date. However, in experiments in which reaction time differences between stimulus types were sufficiently large that such violations might have been expected, typically only few (3-4) subjects participated, and for each subject, relatively few (less than 100) trials were collected for each combination of signal lag and stimulus type. The failure to find evidence for violations of stimulus-type invariance in these experiments may therefore have been due to a lack of power.

The task in the present experiment consisted of a linelength discrimination with two levels of discriminability that produced a substantial reaction time difference. To enhance power, 5 subjects were used, and each subject contributed 300 trials for each combination of stimulus type (easy vs. difficult discrimination) and signal lag.

Simulations 1 and 2 revealed an interesting feature of the pure-guess model regarding the relation between microtradeoffs and macrotradeoffs between guessingcompletion time and guessing accuracy. Whereas the macrotradeoffs were strictly positive, the microtradeoffs changed from being positive at short signal lags to negative at intermediate and long lags. It is not clear how the sophisticated-guessing model might explain such a relation between the tradeoffs. According to the latter model, guessing accuracy is determined by the partial output accumulated by regular processes at the time that the guess response is selected. The model does not necessarily predict that the microtradeoffs should line up with the macrotradeoff, because the variability in guessingcompletion times present in microtradeoffs might reflect variability in the duration of postdecisional guessing processes, such as those involved in executing the guess response. In that case, guessing accuracy could remain largely unaffected by the variation in guessing-completion time present in microtradeoffs. It is difficult to see, however, how the sophisticated-guessing model could explain negative microtradeoffs. Even if such an explanation could be found, the model would still need to explain why the microtradeoff appears to change from being positive at short signal lags to negative at longer lags. It seems reasonable, then, to conclude that an observed pattern of microtradeoffs consistent with that predicted by the pureguess model would provide strong support for that model. 


\section{Method}

Subjects. Five subjects ( 2 male; age range, 19-22) participated in return for payment. They had normal or corrected-to-normal vision and hearing. Each subject participated in six sessions of about $1 \mathrm{~h}$. The subjects were paid $\$ 2$ per hour, plus a bonus based on performance.

Apparatus and Stimuli. The visual stimuli were presented on Seiko color monitors controlled by IBM-compatible microcomputers (equipped with VGA graphics, providing a display resolution of $640 \times 480$ pixels). The visual stimuli were red or brown horizontal lines of three different lengths. The short lines were 60 pixels long (subtending a visual angle of approximately $2.9^{\circ}$ at a typical viewing distance of $50 \mathrm{~cm}$ ), and the long lines were 120 pixels long. The intermediate line length was variable and determined separately for each subject, as explained below. On each trial, a red and a brown line of different lengths were presented symmetrically above and below a central fixation dot at a vertical separation of about $1.0^{\circ}$. On easy trials, one line was long and the other short; on difficult trials, one line was long and the other was of intermediate length. As explained below, the two lines were overlapped by a visual noise mask consisting of a variable number of randomly located red and brown pixels. The mask was centered on the fixation dot and subtended horizontal and vertical angles of $11.5^{\circ}$ and $2.4^{\circ}$, respectively.

The response signal for the signal trials was a $1000-\mathrm{Hz}$ tone of 100 -msec duration, presented through the speaker of the microprocessor.

Responses were made by pressing the " $Z$ " key on the keyboard when the longer line was red and by pressing the "./" key if it was brown.

Design. The experiment included 6 sessions per subject conducted within 2 weeks; Sessions 1-3 served as practice. Each session included 25 blocks with $\mathbf{4 0}$ trials per block. The subjects worked exclusively on the length discrimination task in the first practice session. In the next two practice sessions, the subjects were introduced to the response signal procedure. On each trial block in this procedure, there were 16 regular trials and 8 signal trials at each of three equally probable signal lags $(50,175$, and $300 \mathrm{msec})$. The different trial types occurred in a random order, and the assignment of stimulus types (easy/difficult discrimination) and required response to each trial type was completely counterbalanced. The final three sessions were test sessions. For each subject and each stimulus type, the test sessions yielded overall totals of 600 regular trials and 300 signal trials at each signal lag.

Procedure. Each trial started with the presentation of a central fixation dot, followed at $500 \mathrm{msec}$ by the presentation of the visual stimulus, in the form of two horizontal lines overlapped by a visual noise mask. On signal trials, the response signal was presented at the appropriate lag after onset of the visual stimulus. The visual stimulus remained on for $1,000 \mathrm{msec}$ or until a response had occurred, whichever was longer. A blank interval of $1,000 \mathrm{msec}$ separated each trial from the next.

Two tracking procedures were used to control the performance of each subject in order to place the response signals appropriately with respect to the regular-trial reaction time distributions. The first tracking procedure was designed to result in a median reaction time of 450 msec on easy regular trials. This procedure operated by varying the number of pixels in the visual mask that overlapped the two lines in each visual stimulus, adding 20 pixels if the easy regular response time was less than $450 \mathrm{msec}$ and subtracting 20 pixels if the response exceeded $450 \mathrm{msec}$. The second tracking procedure was designed to produce a median reaction time of $550 \mathrm{msec}$ on difficult regular trials. In this procedure, the intermediate line length varied, increasing by 1 pixel if the difficult regular response time was less than $550 \mathrm{msec}$ and decreasing by 1 pixel if the response exceeded $550 \mathrm{msec}$. Both procedures were very successful in achieving their respective aims.

The subjects were instructed to respond as quickly as possible to the visual stimulus, without committing too many errors. After an error or a late response (over $2,000 \mathrm{msec}$ ), the message "Error or Too Late" appeared on the screen, remaining there until the subject initiated the next trial by pressing one of the keys. In the case of a response signal, the subjects were instructed to respond immediately, provided that they had not responded already, making a best guess about the correct response. No error feedback was provided on signal trials, but the message "Late" appeared on the screen for $1,000 \mathrm{msec}$ if the response occurred later than $300 \mathrm{msec}$ after onset of the response signal.

A bonus system was used to encourage the subjects to follow these instructions. On regular trials, 0.5 cent was earned for a correct response no slower than $500 \mathrm{msec}$ and 0.5 cent was lost for a slower correct response; 3 cents were lost for every incorrect or late response. On signal trials, 0.5 cent was earned for a correct and 0.5 cent was lost for an incorrect response; in addition, 0.75 cent was won if the response occurred within $300 \mathrm{msec}$ after onset of the response signal, and 1.5 cents were lost otherwise. After each block, the bonus earned during that block was displayed with the total bonus earned so far in the session. The subjects earned an average bonus of about $\$ 3.50$ per session.

Simulation. The pure-guess model was fitted to the results from this experiment, according to the same procedure as that used in Simulations 1 and 2 but without averaging across stimulus types. The parameters of the convolution of normal and exponential distributions used in fitting the empirical distribution function of regulartrial reaction times for easy discriminations were $412 \mathrm{msec}$ for the mean and $44 \mathrm{msec}$ for the standard deviation of the normal, and $51 \mathrm{msec}$ for the time constant of the exponential distribution; the corresponding values for difficult discriminations were 462,68 , and $188 \mathrm{msec}$. The corresponding parameters used in fitting the guessing-duration distribution function were 227,23 , and $29 \mathrm{msec}$; the function for difficult discriminations at the shortest signal lag was used to estimate the latter parameters. The moderate speed-accuracy tradeoff observed on regular trials was incorporated by using correction factors of 0.071 and 0.030 for easy and difficult discriminations, respectively (see note 4 ). The parameters of the pure-guess model that provided the best fit to the estimated guessing accuracies for both easy and difficult trials were 0.23 for the maximum facilitation factor and $\mathbf{- 0 . 0 2}$ for the correlation between regular and guess responses.

\section{Results}

Responses on signal trials that occurred later than 350 msec after onset of the response signal were excluded from the analyses (less than 1\%).

Regular and signal trials. In Table 7, the empirical and simulated median reaction times for correct and incorrect responses, and the response accuracy, are shown as a function of stimulus type and signal lag. Discriminability produced a highly significant reaction time effect on regular trials $[F(1,4)=1,269, p<.001]$, close to the 100 -msec difference that the tracking procedures were designed to produce. Significantly more errors were made when the discrimination was relatively difficult $[F(1,4)$ $=25.1, p<.01]$. As was expected, response accuracy on signal trials increased with signal lag $[F(2,8)=171$, $p<.001]$. Response accuracy on signal trials was significantly better for easier discriminations $[F(1,4)=149$, 
Table 7

Experiment 2: Results For the Regular and Signal Trials

\begin{tabular}{|c|c|c|c|c|c|c|}
\hline \multirow{3}{*}{$\begin{array}{c}\text { Stimulus } \\
\text { Type }\end{array}$} & \multicolumn{4}{|c|}{$\begin{array}{c}\text { Median RT } \\
\text { (in msec) }\end{array}$} & \multirow{2}{*}{\multicolumn{2}{|c|}{$\begin{array}{c}\text { Response } \\
\text { Accuracy } \\
\text { (\% Correct) }\end{array}$}} \\
\hline & \multicolumn{2}{|c|}{$\begin{array}{c}\text { Correct } \\
\text { Responses }\end{array}$} & \multicolumn{2}{|c|}{$\begin{array}{c}\text { Incorrect } \\
\text { Responses }\end{array}$} & & \\
\hline & Emp & Sim & Emp & Sim & Emp & Sim \\
\hline \multicolumn{7}{|c|}{ Regular Trials } \\
\hline $\begin{array}{l}\text { Easy } \\
\text { Difficult }\end{array}$ & $\begin{array}{l}455 \\
558\end{array}$ & $\begin{array}{l}455 \\
556\end{array}$ & $\begin{array}{l}427 \\
534\end{array}$ & $\begin{array}{l}428 \\
534\end{array}$ & $\begin{array}{l}94.1 \\
91.6\end{array}$ & $\begin{array}{l}94.1 \\
91.6\end{array}$ \\
\hline \multicolumn{7}{|c|}{ Short-Signal Trials } \\
\hline $\begin{array}{l}\text { Easy } \\
\text { Difficult }\end{array}$ & $\begin{array}{l}300 \\
292\end{array}$ & $\begin{array}{l}297 \\
295\end{array}$ & $\begin{array}{l}300 \\
294\end{array}$ & $\begin{array}{l}292 \\
294\end{array}$ & $\begin{array}{l}55.2 \\
49.7\end{array}$ & $\begin{array}{l}54.9 \\
51.3\end{array}$ \\
\hline \multicolumn{7}{|c|}{ Medium-Signal Trials } \\
\hline $\begin{array}{l}\text { Easy } \\
\text { Difficult }\end{array}$ & $\begin{array}{l}385 \\
404\end{array}$ & $\begin{array}{l}384 \\
406\end{array}$ & $\begin{array}{l}382 \\
407\end{array}$ & $\begin{array}{l}394 \\
413\end{array}$ & $\begin{array}{l}80.2 \\
60.5\end{array}$ & $\begin{array}{l}79.7 \\
62.0\end{array}$ \\
\hline \multicolumn{7}{|c|}{ Long-Signal Trials } \\
\hline $\begin{array}{l}\text { Easy } \\
\text { Difficult }\end{array}$ & $\begin{array}{l}444 \\
487\end{array}$ & $\begin{array}{l}435 \\
493\end{array}$ & $\begin{array}{l}448 \\
501\end{array}$ & $\begin{array}{l}440 \\
519\end{array}$ & $\begin{array}{l}92.5 \\
78.7\end{array}$ & $\begin{array}{l}92.4 \\
77.8\end{array}$ \\
\hline
\end{tabular}

Note-Emp $=$ empirical results. Sim $=$ simulation results. $p<.001]$, and this difference was larger at longer signal lags, as evidenced by a significant interaction of discriminability and signal lag $[F(2,8)=13.5, p<.01]$. Finally, Table 7 demonstrates a good fit between the empirical and the simulation results $\left[\chi^{2}(6)=.33, p>.999\right.$, for correct response latencies; $\chi^{2}(6)=1.24, p>.97$, for incorrect latencies; $\chi^{2}(4)=.089, p>.999$, for overall response accuracy].

More details regarding the reaction times on regular and signal trials are provided in Figure 5, where empirical and simulated distribution functions of regular-trial reaction time (top panel) and signal-trial reaction times (middle panel) are shown as a function of stimulus type and signal lag. As can be seen from this figure, the more difficult discrimination produced not only an overall shift of the regular-trial distribution function to the right but also resulted in a markedly more skewed distribution. The distribution functions of signal-trial reaction times in Experiment 2 are similar to those presented in Figure 3, with largely parallel functions at the different signal lags, except for the lower tails at the longest lag. The overall fit

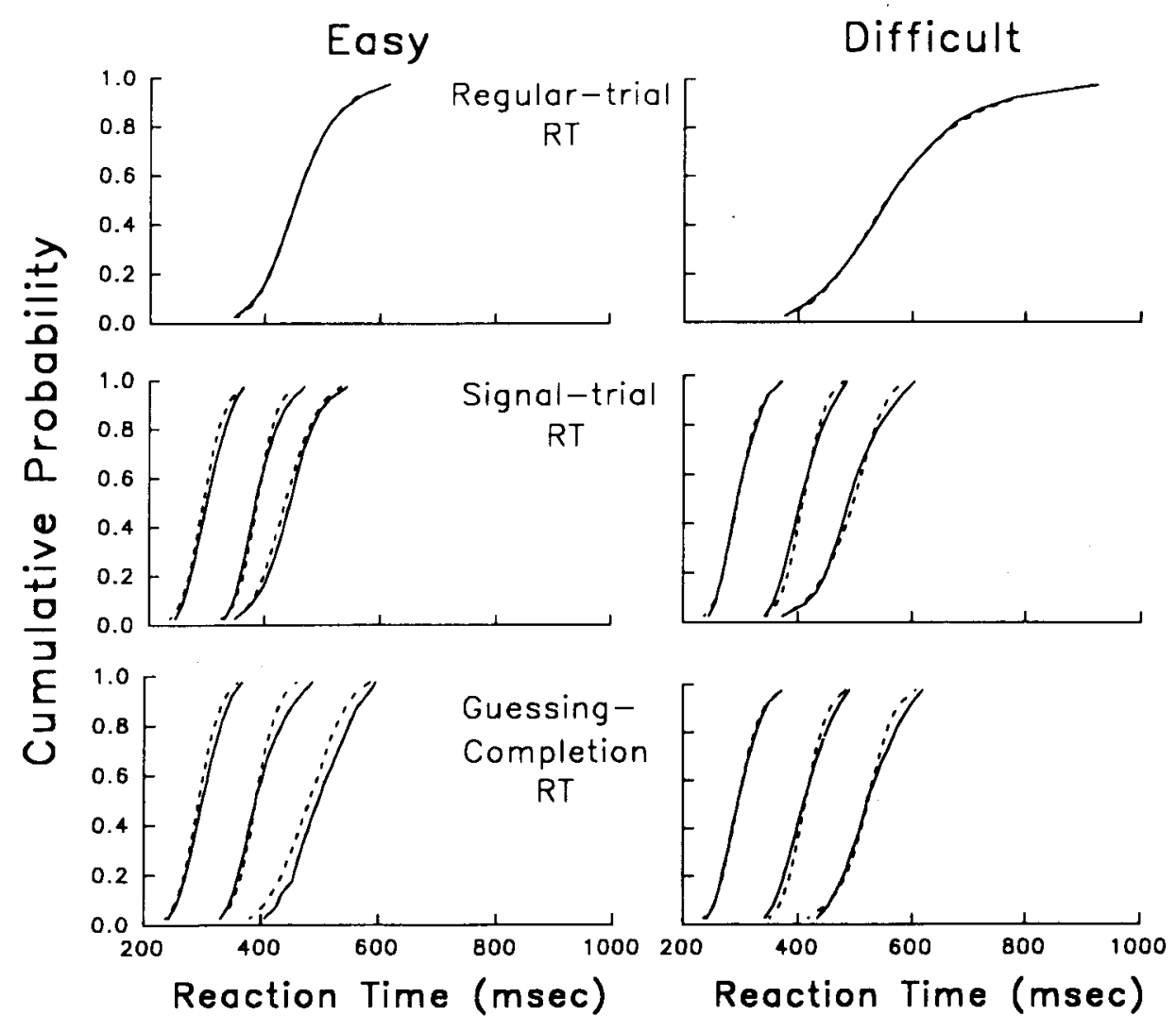

Figure 5. Cumulative distribution functions in Experiment 2, at the lef for easy and at the right for difficult discriminations. The top, middle, and bottom panels illustrate, respectively, the reaction times (RTs) on regular trials, the reaction times on signal trials, and the derived guessing-completion times. Solid lines: empirical results; dashed lines: simulation results. 
Table 8

Experiment 2: Results Regarding the Guessing Processes on Signal Trials

\begin{tabular}{|c|c|c|c|c|c|c|}
\hline \multirow{2}{*}{$\begin{array}{c}\text { Stimulus } \\
\text { Type }\end{array}$} & \multicolumn{2}{|c|}{$\begin{array}{c}\text { Duration } \\
\text { (in msec) }\end{array}$} & \multicolumn{2}{|c|}{$\begin{array}{c}\text { Interquartile } \\
\text { Range } \\
\text { (in msec) } \\
\end{array}$} & \multicolumn{2}{|c|}{$\begin{array}{c}\text { Response } \\
\text { Accuracy } \\
\text { (\% Correct) }\end{array}$} \\
\hline & Emp & Sim & Emp & $\operatorname{Sim}$ & Emp & Sim \\
\hline \multicolumn{7}{|c|}{ Short-Signal Trials } \\
\hline Easy & 249 & 243 & 48 & 41 & 55.3 & 55.1 \\
\hline Difficult & 244 & 244 & 48 & 44 & 49.8 & 51.5 \\
\hline \multicolumn{7}{|c|}{ Medium-Signal Trials } \\
\hline Easy & 216 & 215 & 57 & 47 & 77.6 & 77.3 \\
\hline Difficult & 234 & 236 & 60 & 44 & 58.8 & 59.5 \\
\hline \multicolumn{7}{|c|}{ Long-Signal Trials } \\
\hline Easy & 197 & 182 & 78 & 76 & 91.8 & 91.8 \\
\hline Difficult & 218 & 221 & 74 & 57 & 73.3 & 70.7 \\
\hline
\end{tabular}

Note-Emp $=$ empirical results. Sim $=$ simulation results.

to the empirical signal-trial distribution functions was excellent $\left[\chi^{2}(120)=14.3, p>.999\right]$.

Guessing accuracy and duration. Estimated empirical and simulated distribution functions of guessingcompletion times are shown in Figure 5 (bottom panel) as a function of stimulus type and signal lag. The simulated functions appear to fit the empirical ones well $\left[\chi^{2}(120)=27.6, p>.999\right]$, although there appear to be some systematic deviations in the right tail of the distributions. Also, for easy discriminations, the simulated function at the longest signal lag appears to be shifted to the right by about $20 \mathrm{msec}$, relative to the corresponding empirical function.

Estimated empirical and simulated guessing accuracies and medians and interquartile ranges of the guessingduration distributions are shown in Table 8 , as a function of stimulus type and signal lag. As expected, guess- ing accuracies increased as signal lag increased $[F(2,8)=$ $95, p<.001]$, and they were higher for easy discriminations $[F(1,4)=135, p<.001]$; the interaction of stimulus type and signal lag was also significant $[F(2,8)=$ $6.5, p<.025]$. Interestingly, the estimated guessing accuracy for easy discriminations well exceeded $90 \%$ at the longest signal lag, closely approaching the average accuracy on regular trials.

Median guessing duration showed a strong decrease as signal lag increased $[F(2,8)=62.3, p<.001]$. Most important for the present purposes, guessing durations were significantly shorter for easy discriminations $[F(1,4)=$ $26.7, p<.01]$, and this difference was larger at the longer signal lags $[F(2,8)=12.3, p<.01]$. These results represent a clear violation of stimulus-type invariance of the distribution of guessing durations.

Replicating the results reported by Meyer et al. (1988, Experiment 5), the interquartile range of the distribution of guessing durations showed a marked effect of signal lag in a direction opposite that of the effect on the median $[F(2,8)=61.4, p<.001]$. No other effect on interquartile range approached significance.

The simulation provided reasonably good fits for median guessing duration and guessing accuracy $\left[\chi^{2}(6)=\right.$ $1.45, p>.96$, for median guessing duration; $\chi^{2}(4)=$ $.182, p>.99$, for guessing accuracy]. The simulation did not exhibit the same strong increase in interquartile range as a function of signal lag that was present in the empirical results for difficult discriminations, resulting in a significant difference between empirical and simulated interquartile ranges $\left[\chi^{2}(6)=14.9, p<.05\right]$.

"Empirical" and simulated speed-accuracy microtradeoffs for guess responses are shown in Figure 6 as a function of stimulus type and signal lag. The simulated microtradeoffs exhibit the same pattern found in the previous simulations, being positive at short signal lags and

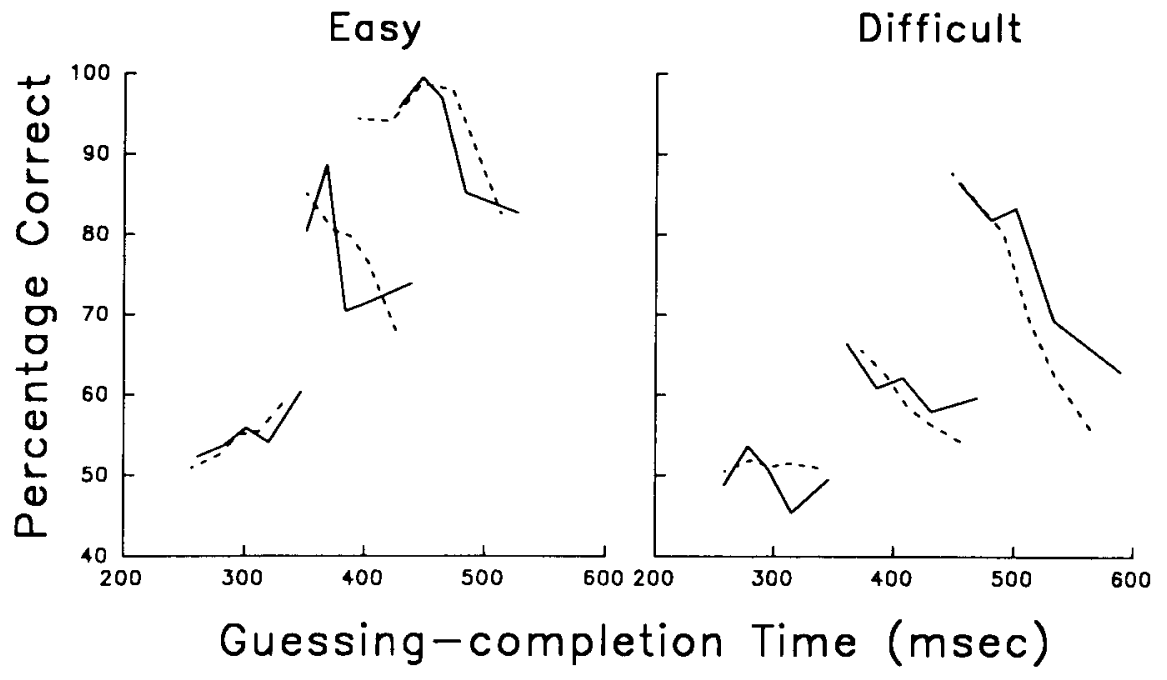

Figure 6. Speed-accuracy microtradeoffs for guess responses in Experiment 2, at the left for easy and at the right for difficult discriminations. Tradeoffs for guess responses at each of the response signal lags are shown, arranged from lower left to upper right in order of ascending signal lag. Solid lines: empirical results; dashed lines: simulation results. 
increasingly negative at intermediate and long signal lags. The empirical microtradeoffs are somewhat more noisy, but it is evident that they exhibit substantially the same pattern as the simulated tradeoffs. In particular, in the one case in which the simulated tradeoff was positive, the empirical tradeoff was positive as well, and in at least three of the four cases in which the simulated tradeoff was negative, the empirical tradeoff was negative as well. The overall fit between empirical and simulated tradeoffs was very $\operatorname{good}\left[\chi^{2}(28)=7.9, p>.999\right]$. Thus, the pure-guess model appears to be able to account not only for the "observed" speed-accuracy macrotradeoff for guess responses, but for the microtradeoffs as well.

\section{Discussion}

Experiment 2 provided two important results. First, a highly significant violation of stimulus-type invariance of the estimated distribution of guessing durations was obtained. This result provides strong evidence that the temporal independence assumption of the speed-accuracy decomposition technique was violated in this experiment, and it cannot be explained by current versions of the sophisticated-guessing model. Why was evidence for violations of stimulus-type invariance not found in previous experiments? One reason may be that these experiments did not provide sufficient power to demonstrate such violations. A second and related reason may be that such violations were especially pronounced in Experiment 2 . As can be seen from the top panel of Figure 5, the distribution of regular-trial reaction times for difficult discriminations is not only shifted to the right relative to the distribution for easy discriminations, but it is also significantly broader and much more skewed to the right. All these factors contributed to a very sizable difference between the hazard functions for easy and difficult discriminations. Earlier, the relative number of spurious guesses was shown to be roughly proportional to the hazard function of the regular-trial reaction times. Thus, a substantially larger relative number of spurious guesses should be expected for easy discriminations, in accordance with the observed higher guessing accuracies and smaller guessing durations for such discriminations. In comparison, in none of the previous experiments did different stimulus types give rise to such dramatically different hazard functions. The procedure in the present experiment thus seems to have created a situation that was particularly well suited for obtaining evidence of possible violations of stimulus-type invariance.

The second important finding in this experiment consisted of the negative speed-accuracy microtradeoffs for guess responses at medium and long signal lags. Such tradeoffs are consistent with the pure-guess model but cannot be easily accounted for by the sophisticated-guessing model.

This experiment replicated the results reported by Meyer et al. (1988, Experiment 5), in that signal lag produced sizable but opposite effects on the median and on the interquartile range of the estimated distribution for guess responses. Again, these results are consistent with the pure-guess model, but it is not clear whether and how they may be accounted for by the sophisticated-guessing model.

In summary, the present experiment has provided further support for the pure-guess model. These and previous results appear to establish the pure-guess model as a reasonable and viable alternative model for the results from speed-accuracy decomposition.

\section{GENERAL DISCUSSION}

The pure-guess model holds that guessing processes have no access to preliminary, partial processing products of regular processes, so that guesses must be pure guesses at chance accuracy. It attributes the estimated abovechance guessing accuracies in several applications of the speed-accuracy-decomposition technique to violations of temporal independence of regular and guess responses-in particular, to facilitating effects of the response signal on the speed of regular responses.

In assuming that guessing processes-or, more specifically, output processes-do not have access to partial information accumulated in prior stages of processing, the pure-guess model is consistent with a class of models of the human information processing system known as discrete models. According to discrete models, information may accumulate continuously within processing stages, but it is transferred to other stages only if it exceeds a minimal amount or grain size (Miller, 1988). In some models, perceptual information is assumed to be made available to output processes in reaction-time tasks only in the form of discrete or categorical codes (see, e.g., Miller, 1982). Related ideas can be found in recent models of selective attention, in which one of the primary functions of attentional mechanisms is to categorize the sensory evidence regarding elements in the visual field; only the resulting discrete perceptual categorizations are assumed to be available or useful to output processes (Bundesen, 1990; Duncan \& Humphreys, 1989). Continuous models, in contrast, assume that a processing stage will transmit whatever amount of information it has accumulated to all the stages to which it is connected (Eriksen \& Schultz, 1979; McClelland, 1979). Discrete models have been criticized on a number of grounds (e.g., by Coles, Gratton, Bashore, Eriksen, \& Donchin, 1985; Eriksen \& Schultz, 1979; and McClelland, 1979) but, as Miller (1988) argues, such criticisms have been based on evidence that is not necessarily inconsistent with discrete models. Evidence in favor of a discrete relation between output stages and prior processing stages has recently been obtained by De Jong, Wierda, Mulder, and Mulder (1988). The results of the present study may be viewed as providing further evidence in favor of discrete models.

\section{Tests and Limitations of the Pure-Guess Model}

The pure-guess model has only two free parameters at its disposal-the facilitation factor, and the correlation of 
regular and guess responses-to account for the observed distributions of signal-trial reaction times, the estimated distributions of guessing durations, and estimated guessing accuracies. This makes it relatively easy to test the model against an available set of results from speed-accuracy decomposition-for instance, with simulations such as those described in the present paper.

A few precautions have to be taken into account for such tests of the model to be valid and useful. The pure-guess model assumes that actual guess responses are at chance accuracy. This feature of the model precludes its application to results from experiments in which this assumption is not warranted. An example of such a case is the study in which Kounios et al. (1987) examined the time course of processing in a sentence-verification task. The very slight effects of signal lag on mean guessing duration, in combination with the very high guessing accuracies found in their study, indicate that these results cannot be fully explained by the pure-guess model. The observed guessing accuracies must therefore be assumed to reflect, at least in large part, the use of partial information in producing a guess response. However, the nature of such partial information in the Kounios et al. study is uncertain. Subjects were asked to judge the truth of sentences such as "All collies are dogs," in which one noun was the name of a natural category and the other the name of an exemplar of a natural category. Importantly, with the set of sentences used in this study, "false" responses were twice as likely as "true" responses, and in relation to this, the relative abstractness of the subject term (category or exemplar) provided a cue about the correct response to a given sentence. Note that the unequal response probabilities and the fact that the relative abstractness of the subject term by itself provided response information are consequences of characteristics of the stimulus material used. These types of information are qualitatively different from the information regarding the semantic relationship between subject and object term on which regular responses would presumably be based. It is partial information of the latter kind that the pure-guess model assumes not to be available to guessing processes; the model in its present form has no provisions for dealing with other kinds of information that can be used in guessing by virtue of specific characteristics of the stimulus material or unequal response probabilities. For this reason, the pure-guess model in its present form cannot be applied to the results reported by Kounios et al.

A similar limitation of the pure-guess model applies when response biases are evident in regular responses. For example, consider the possibility that in a same-different judgment task, subjects will be biased toward the different response when the presentation of the response signal forces them to guess prematurely. Such a bias would preclude the application of the pure-guess model to same and different trials separately, but the model can still be applied when these trials are averaged. It would be possible to incorporate such response biases in the pureguess model if they could be reliably estimated from the experimental results and were consistent across the different signal lags. In general, however, the pure-guess model should be applied with caution to subsets of experimental results that are related to specific stimulus or response types.

\section{Implications for Speed-Accuracy Decomposition}

The results of the present paper raise questions regarding the applicability and usefulness of the speed-accuracy decomposition technique for investigating the dynamics of human information processing. The pure-guess model holds that guessing accuracy as a function of response signal lag does not reflect the time course of information processing in the primary task but depends solely on the form of the regular-trial reaction time distribution and on the amount of facilitation produced by the response signal. Because this model seems capable of accounting for the result from speed-accuracy decomposition in considerable detail, it is unclear whether and how one might be able to infer processing dynamics from observed guessing accuracies.

The problems for the decomposition technique raised by the present paper can be stated more explicitly if one focuses on the sophisticated-guessing model that forms the basis of the original inferential logic of the technique. As described by Meyer et al. (1988), this model imposes some constraints on the form of the guessing-accuracy function; guessing accuracy should not be below chance, should not exceed the accuracy of regular responses, and should increase monotonically over time. In addition, the model predicts stimulus-type invariance of the distribution of guessing duration. Except for stimulus-type invariance, these constraints are quite weak and of little practical use in testing between alternative models. The present paper adds two further criteria that can be used to evaluate the sophisticated-guessing model. First, the model seems unable to explain negative speed-accuracy microtradeoffs for guess responses. Second, the model has no ready provisions for explaining opposite effects of signal lag on the mean and the interquartile range of the distribution of guessing duration. On all three criteria, stimulustype invariance, microtradeoffs, and opposite effects on mean and interquartile range, the evidence presented in the present paper speaks against the sophisticated-guessing model and in favor of the pure-guess model. These results provide reasons for serious concerns regarding the validity of speed-accuracy decomposition as a technique for investigating the real-time dynamics of human information processing.

It would be premature to pass a definitive judgment on the speed-accuracy decomposition technique on the basis of this study alone, especially when the technique is potentially of great importance and when alternative techniques are sparse and not free of problems either. The present study does, however, indicate the need for a more careful assessment of the strengths and weaknesses of speed-accuracy decomposition in order for results of its application to be interpretable with reasonable confidence. 
In particular, it will be useful to determine the relative applicability of the sophisticated-guessing and the pureguess models across a wider range of informationprocessing tasks, and, as is suggested by the present findings, it will almost certainly be necessary to develop ways to correct the estimated guessing-accuracy function for facilitating effects of the response signal.

\section{REFERENCES}

Bernstein, I. H., Clark, M. H., \& Edelstein, B. A. (1969). Intermodal effects in choice reaction time. Joumal of Experimental Psychology, 81, 405-407.

Bernstein, I. H., Rose, R., \& Ashe, V. (1970). Preparatory state effects in intersensory facilitation. Psychonomic Science, 19, 113-114.

Bundesen, C. (1990). A theory of visual attention. Psychological Review, 97, 523-547.

Coles, M. G. H., Gratton, G., Bashore, T. D., Eriksen, C. W., \& Donchin, E. (1985). A psychophysiological investigation of the continuous flow model of human information processing. Journal of Experimental Psychology: Human Perception \& Performance, 11, 529-553.

DE JONG, R. (1991). The role of preparation in overlapping task performance. Manuscript in preparation.

De Jong, R., Wierda, M., Mulder, G., \& Mulder, L. J. M. (1988). Use of partial information in response processing. Journal of Experimental Psychology: Human Perception \& Performance, 14, 682-692.

Duncan, J., Humphreys, G. W. (1989). Visual search and stimulus similarity. Psychological Review, 96, 433-458.

ERIKSEN, C. W., \& SChULTZ, D. W. (1979). Information processing in visual search: A continuous flow conception and experimental results. Perception \& Psychophysics, 25, 249-263.

Hershenson, M. H. (1962). Reaction time as a measure of intersensory facilitation. Journal of Experimental Psychology, 63, 289-293.

Kounios, J., Osman, A. M., \& Meyer, D. E. (1987). Structure and process in semantic memory: New evidence based on speed-accuracy decomposition. Joumal of Experimental Psychology: General, 10, 3-25.

LUCE, R. D. (1986). Response times: Their role in inferring elementary mental organization. New York: Oxford University Press.

MCClellaNd, J. L. (1979). On time relations of mental processes: A framework for analyzing processes in cascade. Psychological Review, 86, 287-330.

Meyer, D. E., Irwin, D. E., Osman, A. M., Kounios, J. (1988). The dynamics of cognition and action: Mental processes inferred from speed-accuracy decomposition. Psychological Review, 95, 183-237.

MiLLER, J. O. (1982). Discrete versus continuous stage models of human information processing: In search of partial output. Journal of Experimental Psychology: Human Perception \& Performance, 8, 273-296.

MiLleR, J. O. (1988). Discrete and continuous models of human information processing: Theoretical distinctions and empirical results. Acto Psychologica, 67, 191-257.

NAvon, D., \& Gopher, D. (1979). On the economy of the human information processing system. Psychological Review, 86, 214-255.

NICKERSON, R. S. (1973). Intersensory facilitation of reaction time: Energy summation or preparation enhancement? Psychological Review, 80, 489-509.

Niemi, P., NAatanen, R. (1981). Foreperiod and simple reaction time. Psychological Bulletin, 89, 133-162.

Posner, M. I., Nissen, M. J., \& KleIN, R. (1976). Visual dominance: An information-processing account of its origins and significance. Psychological Review, 83, 157-171.

RATCLIFF, R. (1979). Group reaction time distributions and an analysis of distribution statistics. Psychological Bulletin, 86, 446-461.

RatclifF, R. (1988). Continuous versus discrete information processing: Modeling accumulation of partial information. Psychological Review, 95, 238-255.
STERNBERG, S. (1969). The discovery of processing stages: Extensions of Donders' method. In W. G. Koster (Ed.), Antention and performance II (pp. 276-315). Amsterdam: North-Holland.

ToDD, J. W. (1912). Reaction to multiple stimuli. Archives of Psychology, 3 (No. 25). New York.

\section{NOTES}

1. The experiments cited here do not exhaust the available evidence on the effects of signal lag on mean guessing duration. In Experiment 2 of Meyer et al. (1988) and in the experiments reported by Kounios et al. (1987), mean guessing duration was found to be invariant with signal lag. In the former experiment, however, only relatively long signal lags were used, and as will be shown later, it is at relatively short signal lags that effects on mean guessing duration are most apparent. The results reported by Kounios et al. represent a special case that will be discussed separately later.

2. The following example may illustrate the computation of the facilitation factor. Suppose that $70 \%$ of the signal-trial reaction times $\propto c$ curred no later than 300 msec after onset of the accessory stimulus and that the same percentage of regular-trial reaction times was found to occur no later than $375 \mathrm{msec}$ after signal onset. The value of the facilitation factor at $375 \mathrm{msec}$ after signal onset is then computed as $(375-300) / 375=0.20$.

3. The reaction time distribution for regular trials was divided into $1010 \%$ reaction time bins. Error probabilities for responses in each bin were set according to the following equation:

$$
P_{\text {ert }}(i)=P_{\text {err }}(1+k)(11-2 i), \quad i=1, \ldots 10,
$$

where $P_{\text {err }}$ is the overall error probability and $k$ is a constant. The value of $k$ was determined so that the median reaction times for incorrect and correct regular-trial responses in the simulations were close to the corresponding empirical values ( $k$ is 0.042 in Simulation 1 and 0.045 in Simulation 2).

4. The procedure for obtaining a preset correlation between regular and guessing responses will be illustrated for a value of 0.25 . After two random samples were drawn from the two distributions, the current correlation was computed. Two new random samples were drawn if the current correlation was less than 0.25 ; resampling was repeated until this correlation was at least $\mathbf{0 . 2 5}$. Due to the tendency of the correlation to drift back toward $\mathbf{0}$ with random sampling, this procedure resulted in a correlation that was very close to the desired value throughout the simulation.

5. The correctness of the simulation program was verified in separate simulation runs in which the correlation between regular and guessing responses and the maximum facilitation factor were set to zero. These simulations produced, as required, estimated guessing accuracies of $50 \%$ and estimated guessing duration distributions that exactly matched the original distributions.

6. All empirical results were averaged across stimulus type. Such averaging was necessary to enhance the stability of these results. For instance, Meyer et al. (1988, Table 9) reported highly nonmonotonic guessing-accuracy functions for some of the individual stimulus types. Nonmonotonic guessing-accuracy functions are inconsistent with either the sophisticated-guessing model or the pure-guess model and most likely indicate that the results for individual stimulus types were rather unstable.

\section{Appendix A \\ Hazard Function and Relative Proportion of Spurious Guesses}

To derive the relationship between the hazard function of the regular-trial reaction time distribution and the relative number of spurious guesses, two simplifying assumptions are made. First, guessing reaction time is assumed to be constant $\left(T_{\mathrm{s}}\right)$. Second, facilitation is assumed to speed up all regular responses that occur after presentation of the response signal by a constant amount of time $\left(T_{f}\right)$. 
Assuming temporal independence, the total proportion of guesses for a given signal lag $\left(T_{1}\right)$ is given by

$$
P\left(\text { guess } \mid T_{1}\right)=1-F\left(T_{1}+T_{\mathrm{g}}\right) \text {, }
$$

where $F(t)$ is the distribution function of regular-trial reaction times and $\left(T_{1}+T_{\mathrm{g}}\right)$ is the (constant) guessing-completion time. With facilitation, the total proportion of spurious guesses is given by

$$
P\left(\text { spurious } \mid T_{\mathrm{l}}\right)=\int_{T_{1}+T_{\mathrm{l}}}^{T_{1}+T_{\mathrm{l}}+T_{\mathrm{f}}} f(t) d t,
$$

where $f(t)$ is the probability density function of regular-trial reaction times. The relative proportion of spurious guesses is thus given by

$$
\begin{aligned}
\int_{T_{\mathrm{l}}+T_{\mathrm{g}}}^{T_{1}+T_{\mathrm{g}}+T_{\mathrm{f}}} f(t) & d t /\left[1-F\left(T_{1}+T_{\mathrm{g}}\right)\right] \\
& \approx T_{\mathrm{f}}\left\{f\left(T_{1}+T_{\mathrm{g}}\right) /\left[1-F\left(T_{1}+T_{\mathrm{g}}\right)\right]\right\},
\end{aligned}
$$

where the latter approximation holds if $T_{\mathrm{f}}$ is small relative to the rate of change of $f(t)$.

From Equation $\mathrm{Al}$, the relative proportion of spurious guesses can be seen to be approximated by the product of the strength of the facilitating effect, which is assumed to be independent of signal lag, and the value of the hazard function of the regulartrial reaction time distribution at guessing-completion time. Thus, the relative proportion of spurious guesses will increase with signal lag whenever the hazard function of the regular-trial reaction time distribution is strictly increasing.

\section{Appendix B \\ Derivation of the Speed-Accuracy Microtradeoff for Guess Responses}

All derivations presented here are based on the assumption of the parallel sophisticated-guessing model that regular and guessing processes operate independently on signal trials, and that the response on such trials is produced by the processes that finish first. As shown by Meyer et al. (1988), the following relation holds between the cumulative distribution functions of guessing-completion times $\left[F_{\mathrm{g}}(C)\right]$, signal-trial reaction times $\left[F_{\mathrm{s}}(C)\right]$, and regular-trial reaction times $\left[F_{\mathrm{r}}(C)\right]$

$$
F_{\mathrm{g}}(C)=\left[F_{\mathrm{s}}(C)-F_{\mathrm{r}}(C)\right] /\left[1-F_{\mathrm{r}}(C)\right],
$$

where $C \geq 0$.

A correct signal-trial response is produced either by regular processes that finish first and produce a correct response, or by guessing processes that finish first and produce a correct response. The probability of a correct signal-trial response that occurs no later than a constant time $C$ after onset of the test stimulus is therefore given

$$
\begin{aligned}
P\left(\text { correct and } t_{\mathrm{s}} \leq C\right)= & P\left(\text { correct and } t_{\mathrm{r}} \leq t_{\mathrm{g}} \text { and } t_{\mathrm{r}} \leq C\right) \\
& +P\left(\text { correct and } t_{\mathrm{g}}<t_{\mathrm{r}} \text { and } t_{\mathrm{g}} \leq C\right)
\end{aligned}
$$

where $C \geq 0, t_{s}$ is the random signal-trial reaction time, and $t_{\mathrm{r}}$ and $t_{\mathrm{g}}$ are the random completion times of the regular and guessing processes, respectively. The second term on the right of Equation B2 denotes the probability that guessing processes finish first and no later than $C$, and produce a correct response. This probability is a function of $C$ and will be denoted by $Y(C)$. The first term on the right of Equation B2 denotes the probability that regular processes finish before guessing processes and no later than $C$, and produce a correct response. This term can be rewritten as

$$
\begin{aligned}
& P\left(\text { correct } \mid t_{\mathrm{r}} \leq t_{\mathrm{g}} \text { and } t_{\mathrm{r}} \leq C\right) P\left(t_{\mathrm{r}} \leq t_{\mathrm{g}} \text { and } t_{\mathrm{r}} \leq C\right) \\
& =\int_{0}^{\infty} P\left(\text { correct } \mid t_{\mathrm{r}} \leq \min [C, x]\right) f_{\mathrm{g}}(x) d x \int_{0}^{C} f_{\mathrm{r}}(x)\left[1-F_{\mathrm{g}}(x)\right] d x
\end{aligned}
$$

where $P\left(\right.$ correct $\left.\mid t_{\mathrm{r}} \leq \min [C, x]\right)$ is the probability that the regular processes produce a correct response, given that these processes finish no later than the minimum of $C$ and $x$, and $f_{\mathrm{r}}(x)$ and $f_{z}(x)$ are the probability-density functions of the completion times of the regular and guessing processes, respectively.

Substituting Equation B3 into Equation B2 gives

$Y(C)=P\left(\right.$ correct and $\left.t_{\mathrm{s}} \leq C\right)$

$-\int_{0}^{\infty} P\left(\right.$ correct $\left.\mid t_{\mathrm{r}} \leq \min [C, x]\right) f_{\mathrm{B}}(x) d x \int_{0}^{C} f_{\mathrm{r}}(x)\left[1-F_{\mathrm{g}}(x)\right] d x$,

where $C \geq 0$. All terms on the right of Equation B4 can be estimated or computed from the observed error rates and reaction times on the regular and signal trials (Meyer et al., 1988). This equation can therefore be used to estimate the probability of a correct guess response that occurs no later than $C$.

The probability of a guess response, correct or incorrect, that occurs no later than $C$, denoted by $Z(C)$, is given by

$Z(C)=P\left(t_{\mathrm{g}} \leq t_{\mathrm{r}}\right.$ and $\left.t_{\mathrm{g}} \leq C\right)=\int_{0}^{C} f_{\mathrm{g}}(x)\left[1-F_{\mathrm{r}}(x)\right] d x$,

where $C \geq 0$. The average accuracy of guess responses in any interval [ $\left.C_{1}, C_{2}\right]$ with $C_{1} \geq 0$ and $C_{2}>C_{1}$ can then be computed as follows

$$
\begin{aligned}
P\left(\text { correct } \mid t_{\mathrm{g}}<t_{\mathrm{r}}\right. & \text { and } \left.C_{1}<t_{\mathrm{g}}<C\right) \\
& =\left[Y\left(C_{2}\right)-Y\left(C_{1}\right)\right] /\left[Z\left(C_{2}\right)-Z\left(C_{1}\right)\right] .
\end{aligned}
$$

This equation can be used to estimate the speed-accuracy tradeoff for guess responses to any desired degree of precision. 